\title{
Overt and Covert Identification of Fragmented Objects Inferred From Performance and Electrophysiological Measures
}

\author{
Maria Pia Viggiano \\ University degli Studi di Firenze
}

\author{
Marta Kutas \\ University of California, San Diego
}

\begin{abstract}
The authors investigated visual processing leading to object identification by manipulating the number of fragments and nature of the study. During the study, participants either named or drew objects in Experiment 1 and drew them all in Experiment 2. During the test, participants made an identification judgment at each of 6 different fragmentation levels for studied and new objects. Fewer fragments were needed to identify studied than unstudied objects. Reaction times were faster for studied than unstudied objects both at identification and at the preceding level. Event-related brain potentials (ERPs) to unidentified objects were characterized by a late negativity in contrast to a positivity to identified objects. ERPs to studied but not to new objects contained a smaller and later version of the identification positivity at level just prior to identification, which was not due to differential response confidence. Much covert visual analysis and even object identification may precede overt identification, depending on the nature of prior experience.
\end{abstract}

At present, there is no single psychological or neural account of how people identify the objects that they see. Of the handful of different theories of visual object identification that have been proposed, all describe the identification process as some function of the visual input (retinal image) and the structure and content of the processor (memory). The identification of an object is presumed to emerge from a dynamic interplay between bottom-up, data-driven, sensory processes and top-down, conceptually driven, mnemonic processes. The various theories differ primarily in the finer details of how this happens. Although there is a consensus that retinal images must be interpreted, presumably by processes that compare visual input against representations in memory until a match (i.e., identification) emerges, there is less agreement as to either the nature or the number of memory traces involved. In part, this is due to the changing views of what constitutes memory, which now include distinctions between memories that are easy to consciously access and talk about (declarative or explicit memories) and those that manifest their existence through an individual's actions or preferences (procedural or implicit memories).

Other points of contention concern the specifics of the (a) matching process, (b) nature of the sensory representation(s), (c) nature or number of object representations in memory, (d) relative contributions of bottom-up versus

Maria Pia Viggiano, Dipartimento di Psicologia, Universita degli Studi di Firenze, Firenze, Italy; Marta Kutas, Department of Cognitive Science, University of California, San Diego.

The research was supported in part by Grants AG08313 and MH52893 and by a postdoctoral fellowship from the McDonnellPew Cognitive Neuroscience Institute at the University of California, San Diego. The helpful comments of J. Weckerly and the technical assistance of R. Ohst are much appreciated.

Correspondence concerning this article should be addressed to Maria Pia Viggiano, Dipartimento di Psicologia, University degli Studi di Firenze, via San Niccolo 93, 50125 Firenze, Italy. Electronic mail may be sent to mpviggiano@psico.unifi.it. top-down processes, and (e) the time course(s) of their involvement and interactions, if any. For example, it has been variously proposed that sensory input is matched against a bevy of templates, feature lists, or configurations of elemental shapes called geons, among others (Biederman, 1987). There are also various proposals as to the nature of object representations in memory. For example, template theories require that there be a specific memory trace (template) for each and every object that an individual might identify, although other theories rely on fewer and more abstract representations called prototypes (or pictogens; Humphreys \& Bruce, 1989; Warren \& Morton, 1982). On prototype accounts, object identification is an outcome of a match between a specific object token and the representation(s) of its type in memory (Treisman, 1992).

There is also little agreement as to the time courses of various object identification processes (Humphreys \& Riddoch, 1987). At one extreme, object identification is viewed as the outcome of an exhaustive, strictly bottom-up analysis of the current visual input. Less extreme models allow more continuous use of memories during the identification process. In this view, visual identification is not constrained to unfold in a fixed, sequential order (e.g., from physical analyses to categorical and semantic classification to verbal labeling); rather, it is seen as emerging from a cascade of processes wherein high-order stages are engaged before some, although not necessarily all, operations at lower stages are completed.

These fundamental issues concerning visual object identification have long been investigated with incomplete, fragmented, or masked objects. Identifying and naming a fragmented visual pattern involves analysis of the single fragments and their relations, reference to memory for reasonable categorizations, and links to its verbal label. Since the pioneering work of Leeper (1935), it has been known that prior exposure significantly facilitates the identification of fragmented pictures. Effects of leaming and 
memory on the identification of fragmented stimuli have also been observed more recently (Parkin \& Russo, 1990; Snodgrass \& Corwin, 1988; Snodgrass \& Feenan, 1990; Wippich, Mecklenbrauker, Wentura, \& Srumpel, 1991).

Accurate identification requires that an individual know both what an object is and what it is called. Neuropsychological evidence indicates that these processes are dissociable (McCarthy \& Warrington, 1990). After brain damage, some individuals (visual agnosics) display a profound inability to recognize an object for what it is despite normal vision, whereas others (anomics) show by their actions that they know what the object is and what it is typically used for but cannot name it; still others may not be able to use it properly. The tip-of-the-tongue experience attests to a similar dissociation even in intact individuals (Brown \& MacNeill, 1966; Nelson, Gerler, \& Narens, 1984; Valentine \& Moore, 1995). Accordingly, it should not be surprising to find that much covert analysis including identification may precede conscious recognition and overt identification.

To track the time course of these conscious and unconscious processes leading to overt identification, we combined measures of overt behavior (identification accuracy and reaction time [RT] latency) with those of event-related brain potentials (ERPs) recorded prior to, during, and after the identification of an object depicted in a series of fragmented versions. Each object was revealed across six frames, each increasingly less fragmented (more complete) than the previous one up to the complete drawing. We used the ERPs to track brain activity during visual object identification, both on a millisecond by millisecond basis for any given stimulus, as well as on a second by second basis across stimuli both before and after actual object identification. Our primary aim was to explore the time course and quality of the processing of visual input that has heretofore defied detailed investigation, namely that which does not immediately lead to identification.

The amount of visual information was manipulated by gradually and systematically increasing the number of fragments that composed the object to be identified. We manipulated the study by exposing the participant to the complete forms of objects (in one of two study tasks) that they would later have to identify in a less complete form (i.e., more fragmented). We assumed that such exposure would lead to the formation of memory traces whose access and use could be contrasted with those for fragmented objects for which there had been no such recent exposure. Our working hypothesis was that token representations would be used in the identification process whenever they existed (from the study), whereas the identification of fragments of new, unstudied objects would have to rely on previously established prototypic representations in longterm memory, and that visual processing would differ in these two conditions. We anticipated that traces from the study would unarguably serve as more efficient primes, thereby reducing the amount of information necessary for successful identification, and that this might be evident in ERPs to studied items even before they could be overtly identified.

\section{Experiment 1}

Experiment 1 consisted of a study phase followed by an identification phase. During the study, participants were exposed to line drawings of complete objects, which they either named or drew by hand. In the subsequent identification phase, participants were shown ascending sequences of fragmented versions of objects, including those that they had just named or drawn, as well as new ones. Their task was to name the fragmented object if they could. Hence, participants had to integrate fragments into a meaningful perceptual whole, achieving perceptual closure (the phenomenon described by Gestalt theory of filling in gaps in fragmented material and seeing a meaningful form; Koffka, 1935; Mooney, 1954; Snodgrass \& Feenan, 1990). We expected that prior exposure would facilitate subsequent identification of partial fragments of these objects (relative to new ones) regardless of how they were studied (named or drawn). However, we also expected that drawing would lead to greater priming than naming, for several reasons. First, we thought that drawing (relative to naming) would direct more focal attention to the very visual features that would be important for perceptual analysis of the fragments during identification. Second, we believe that there is considerable overlap between the drawing and identification processes (such as perceptual closure) as both rely on the relation among fragments. Third, we figured that at least some of the objects that were drawn were also named (covertly). Fourth, we anticipated that more time would be spent processing the drawn as opposed to the just-named objects. If our assumptions are valid, then within a levels-of-processing framework (Craik \& Lockhart, 1972), objects that were drawn would be processed more deeply, thereby forming a more durable memory trace than those merely named. However, preexposure in either case was expected to prime identification of the fragmented version relative to new objects.

The questions of main interest for Experiment 1 were the extent to which the time course and accuracy of the identification of fragmented objects would be affected by recent exposure to their complete form, and how this interacted with the nature of study (name, draw), or the number of fragments at test, if at all. In addition, we were especially interested in the effects of prior exposure on visual processing that did not immediately lead to identification. Thus, we examined responses both to two-fragment levels prior $(-2)$ and one-fragment level prior $(-1)$ to successful identification (0). In this way we could investigate how memory (prior exposure or not) and sensation (amount of information) interact in the processing of objects that are not yet identifiable. It is in this unique opportunity to examine the ongoing but covert processes prior to identification that the ERP technique is especially informative.

To date, there has been only one published report with ERPs to investigate the recognition and identification of fragmented line drawings (Stuss, Picton, Cerri, Leech, \& Stethem, 1992). Stuss et al. found that ERPs to highly fragmented objects had a large negative component (around $400 \mathrm{~ms}$ ) that was much less pronounced in the ERPs to the less fragmented (more complete) objects. These ERP differ- 
ences were taken as an index of priming by the previous presentation of the complete object. However, as the objects at various levels of fragmentation were presented randomly rather than systematically (as in Snodgrass \& Feenan, 1990), the amount of savings as a function of increasing levels of completeness could not be assessed. Indeed, Stuss et al. admitted that they had not properly controlled the relation between priming and levels of fragmentation; ERPs for one level were averaged without consideration to whether the same drawing had been previously presented at a higher or lower level of fragmentation.

\section{Method}

\section{Participants}

Sixteen right-handed adults ( 9 women) between 19 and 30 years of age, with no left-handed members in their immediate family and with normal or corrected-to-normal vision were paid $\$ 5$ an hour for their participation in a 3 - to 3.5 -hr experiment.

\section{Stimuli}

Stimuli were line drawings of common objects spanning a number of categories (e.g., animals, clothes, vehicles, and tools; Snodgrass, Smith, Feenan, \& Corwin, 1987; Snodgrass \& Vanderwart, 1980). Each drawing was presented on a cathode ray tube under the control of a personal computer and subtended an angle between $5^{\circ}$ and $10^{\circ}$ along both vertical and horizontal dimensions. Two lists of 113 items each were selected from the set of 260 pictures of objects and animals standardized by Snodgrass and Vanderwart (1980). Each list was further divided such that 38, 37, and 38 items were assigned to the name, drawn, and new conditions, respectively. Eight participants received one list of items, and 8 participants received the other list; there were no reliable list effects.

\section{Electrophysiological Recordings}

Electrophysiological recordings were made with tin electrodes embedded in an elastic cap from 19 scalp locations of the International 10-20 System (Jasper, 1958) including frontal (Fz, $\mathrm{Fpl}, \mathrm{Fp} 2, \mathrm{~F} 2, \mathrm{~F} 3, \mathrm{~F} 7$, and F8), central (Cz, C3, and C4), parietal (Pz, P3, and P4), temporal (T3, T4, T5, and T6), and occipital (O1 and $\mathrm{O} 2$ ) sites. An electrode over the left mastoid process was used as a reference during recording. Data were rereferenced off-line to the average of the voltage at left and right mastoids. Recordings between electrodes placed lateral to each eye were used to monitor horizontal eye movements. Recordings from an electrode below the right eye (referred to the left mastoid) were used to monitor vertical eye movements and blinks. These electro-oculographic recordings were used to eliminate artifact-contaminated trials $(\sim 10 \%)$. The electrical activity was amplified with a bandpass of $0.01-100 \mathrm{~Hz}$ and digitized at $250 \mathrm{~Hz}$. ERPs were computed for epochs extending from $200 \mathrm{~ms}$ before picture onset to $1,600 \mathrm{~ms}$ after.

Mean amplitude and peak-to-peak measurements were made within designated latency ranges relative to the average amplitude in the $200 \mathrm{~ms}$ prior to each stimulus. All measurements were submitted to repeated measures analyses of variance (ANOVAs). The Greenhouse-Geisser correction for violations of sphericity was applied to all treatments with more than one degree of freedom in the numerator. The Tukey procedure was used for all post hoc comparisons.

\section{Procedure}

Participants were tested individually in an electrically isolated and sound-attenuating chamber. After electrode application, participants were instructed to minimize muscle tension, eye movements, and blinking during stimulus presentation. Written and oral instructions were given prior to each phase of the experiment.

In the study phase, 75 line drawings of complete objects (Fragmentation Level 8) were presented for $700 \mathrm{~ms}$ each. Between 1,300 to $1,800 \mathrm{~ms}$ before each object, the word name or draw appeared on the screen, informing participants of their task for the upcoming trial: naming the object aloud or drawing the object on a piece of paper for $45 \mathrm{~s}$. Thirty-eight objects were presented for naming and 37 for drawing. During the identification phase, the 75 objects from the study phase and 38 new objects were presented one at a time. Each object was shown in an ascending sequence at six different levels of fragmentation (Levels 2, 3, 4, 5, 6, and 8), proceeding from the most fragmented (Level 2) to the complete version (Level 8; see Figure 1). Each fragmentation level was shown for a duration of $500 \mathrm{~ms}$. Participants were not told that some objects were ones seen during the study. They were asked to press one of two buttons, once to indicate if they did not think that they could identify the object ("certain no" response) or twice if they were certain that they could not identify it ("very certain no" response). Similarly, participants pressed a different button either once if they thought they could identify the object ("certain yes" response) or twice if they were quite certain that they could identify it ("very certain yes" response). Following each affirmative response, participants were asked to name the object and were given feedback on the correctness of their identification. The intertrial interval depended on RT. On average, the identification phase lasted about $1.25 \mathrm{hr}$ within the $3.5-\mathrm{hr}$ experimental session (including electrode preparation and clean up). Note that the entire stimulus sequence was always presented and RTs and ERPs were recorded for each level, regardless of the level at which the entire stimulus sequence was actually identified.

\section{Results}

\section{Study Phase}

ERPs during study were characterized by a negativepositive-negative complex between 100 and $250 \mathrm{~ms}$ followed by a positive wave at about $800 \mathrm{~ms}$ that was especially large at anterior-central leads. These ERPs did not differ significantly (in morphology, component amplitudes, or latencies) as a function of whether the object was named or drawn.

\section{Identification}

Behavioral data. As expected, objects that had been seen previously were identified on the basis of fewer fragments than objects that had not been studied (Gollin, 1960; Snodgrass \& Corwin, 1988). Moreover, RTs were faster for studied than unstudied objects, both at identification and at the immediately preceding fragmentation level.

Accuracy. Mean identification thresholds at the fragmentation level at which the objects were correctly identified on average were $4.8(S E=0.10)$ for drawn objects, 5.3 $(S E=0.09)$ for named objects, and $6.4(S E=0.10)$ for the new objects; main effect of study, $F(2,30)=133.17, p<$ .00001. A Tukey test showed that identification thresholds of 


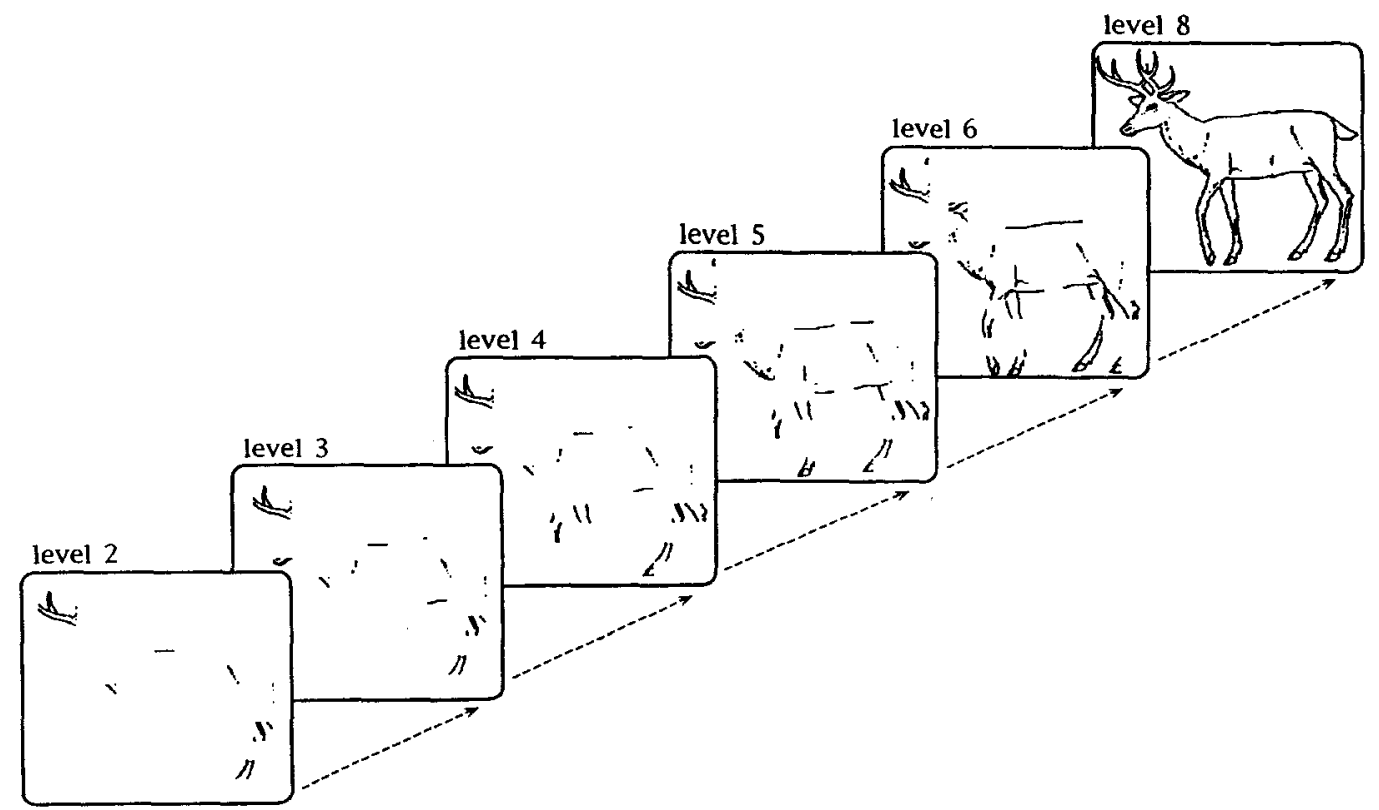

Figure 1. An example of the six levels of fragmentation for one object shown in the order presented during the identification phase. Each object was presented in an ascending sequence from the fewest fragments (Level 2) to the complete object (Level 8); the fragments at each level were presented for a half second.

all three study conditions reliably differed from each other $(\mathrm{p}<.01)$.

RTs. RTs from all 16 participants before, after, and at identification collapsed across response certainty are shown in Figure 2. These data were subjected to a two-way ANOVA

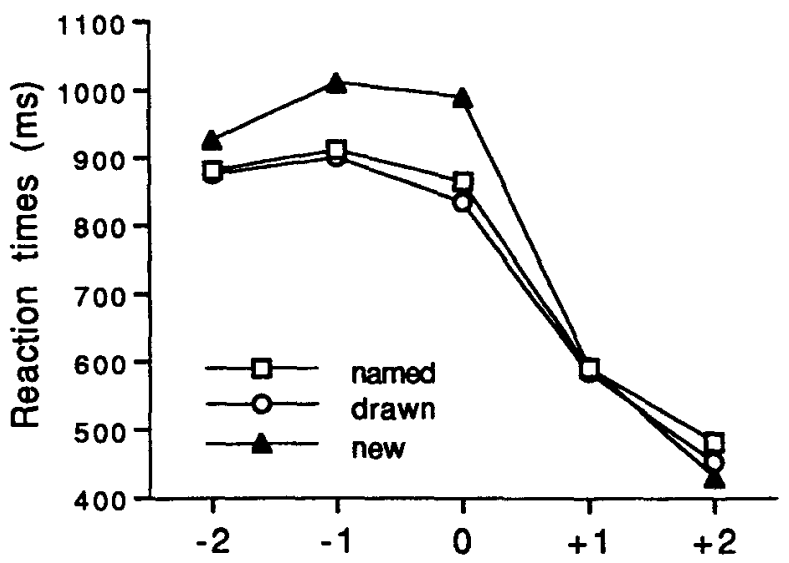

Figure 2. Mean reaction times (RTs) across 16 participants in Experiment 1 to fragments during the time course leading to identification. RTs are shown for comparisons to fragments of objects that had been named (open squares) or drawn (open circles) in the study phase versus those that were new (filled triangles) at five different points in the identification sequence: at identification (0), one and two fragmentation levels before $0(-1$ and -2$)$, and at one and two fragmentation levels after $0(+1$ and +2$)$. Note that because measurements are relative to the fragmentation level at which identification occurs, the RT values at each point are collapsed across several fragment levels. with repeated measures with three levels of study (named, drawn, and new) and five levels of the time course variable defined relative to the level at which identification $(0)$ occurred: $-2,-1,0,+1,+2$. The main effect of the study was significant, $F(2,30)=13.40, p<.0001$. RTs to named and drawn objects did not differ from each other but were significantly faster than those to new objects $(p<.01)$. The main effect of time course was significant, $F(4,60)=42.03$, $p<.00001$, with the fastest RTs to objects after identification. The Study X Time Course interaction also was significant, $F(8,120)=7.98, p<.0001$. Analysis of the simple main effects indicated a significant effect of study on RTs at $-1, F(2,30)=10.73, p<.0001$, and at $0, F(2,30)=$ $22.45, p<.0001$. Both just before and upon identification, fragments of new objects were responded to more slowly than to those of old objects, whether they had been named or drawn. No reliable differences were found at -2 .

Effects of response certainty on accuracy and RTs. At identification (i.e., 0), RTs were faster for studied than unstudied objects and for "very certain yes" than "certain yes" responses (Figure 3). Prior to identification, participants' response certainty did not interact with the study. At -2 , the mean percentages of "very certain no" and "certain no" responses were $56 \%$ and $44 \%$, respectively, $F(1,13)=$ $2.99, p=.10$; at -1 , the percentages of "very certain no" and "certain no" responses were $35 \%$ and $65 \%$, respectively, $F(1,13)=5.12, p<.05$. The Study X Certainty interaction was not significant. At -1, RTs were faster for studied than unstudied pictures. At both -1 and -2 , RTs were faster for "very certain no" than "certain no" responses (Figure 4). 
ERP analyses. In line with Stuss et al. (1992), ERPs to unidentified objects were characterized by a large negativity between 250 to $1,000 \mathrm{~ms}$, whereas those to identified objects were characterized by a large positivity (peaking around 600 $\mathrm{ms}$ or so) in the same latency range (Figure 5). A new finding, however, is the effect of recent exposure on this identification effect. In contrast to the very abrupt transition from negativity prior to identification to positivity upon identification for new fragments (right-hand column), the transition for studied objects (left and middle columns) is more gradual. The primary source of the difference rests in the ERPs to studied objects at -1 ; these contain a small, late positivity compared with ERPs for -2 (shaded area) that is not present for new objects at -1 or -2 . Although smaller and later, this positivity to unidentified fragments is similar to the identification positivity.

We analyzed ERP mean amplitudes between 300-600, 600-1,000, 1,000-1,300, and 1,300-1,600 ms for unidentified and identified objects. A four-way ANOVA with repeated measures was conducted on 15 participants with three levels of study (named, drawn, and new), three levels of time course $(2,-1$, and 0$)$, three levels along the anterior-posterior electrodes (anterior: F7, F3, Fz, F4, and F8; central: T3, C3, Cz, C4, and T4; posterior: T5, P3, Pz, $\mathrm{P} 4$, and T6), and five levels from left-to-right electrodes (left lateral: F7, T3, and T5; left medial: F3, C3, and P3; central: $\mathrm{Fz}, \mathrm{Cz}$, and $\mathrm{Pz}$; right medial: $\mathrm{F} 4, \mathrm{C} 4$, and $\mathrm{P} 4$; right lateral: F8, T4, and P6). The main effect of time course was significant from $300 \mathrm{~ms}$ poststimulus onset on: for 300-600 $\mathrm{ms}, F(2,28)=85.57, p<.00001 ; 600-1,000 \mathrm{~ms}, F(2,28)=$ $104.80, p<.00001 ; 1,000-1,300 \mathrm{~ms}, F(2,28)=37.35, p<$ $.00001 ; 1,300-1,600 \mathrm{~ms}, F(2,28)=7.88, p<.01$. The main effect of study was not reliable anywhere between 300 and 1,600 ms. However, there was a significant three-way interaction between study, time course, and left-to-right electrode between 1,000 and 1,300 ms $(p<.05)$. As can be

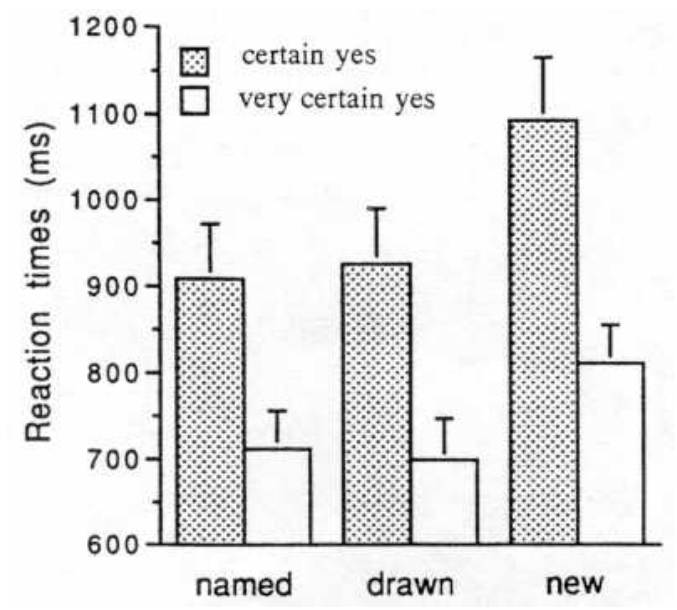

Figure 3. Mean reaction times (RTs; and standard errors) across 13 participants in Experiment 1 for correct identifications during the test phase as a function of response certainty. Speed of "certain yes" and "very certain yes" responses are shown for fragments of objects that had been named, drawn, or were new.
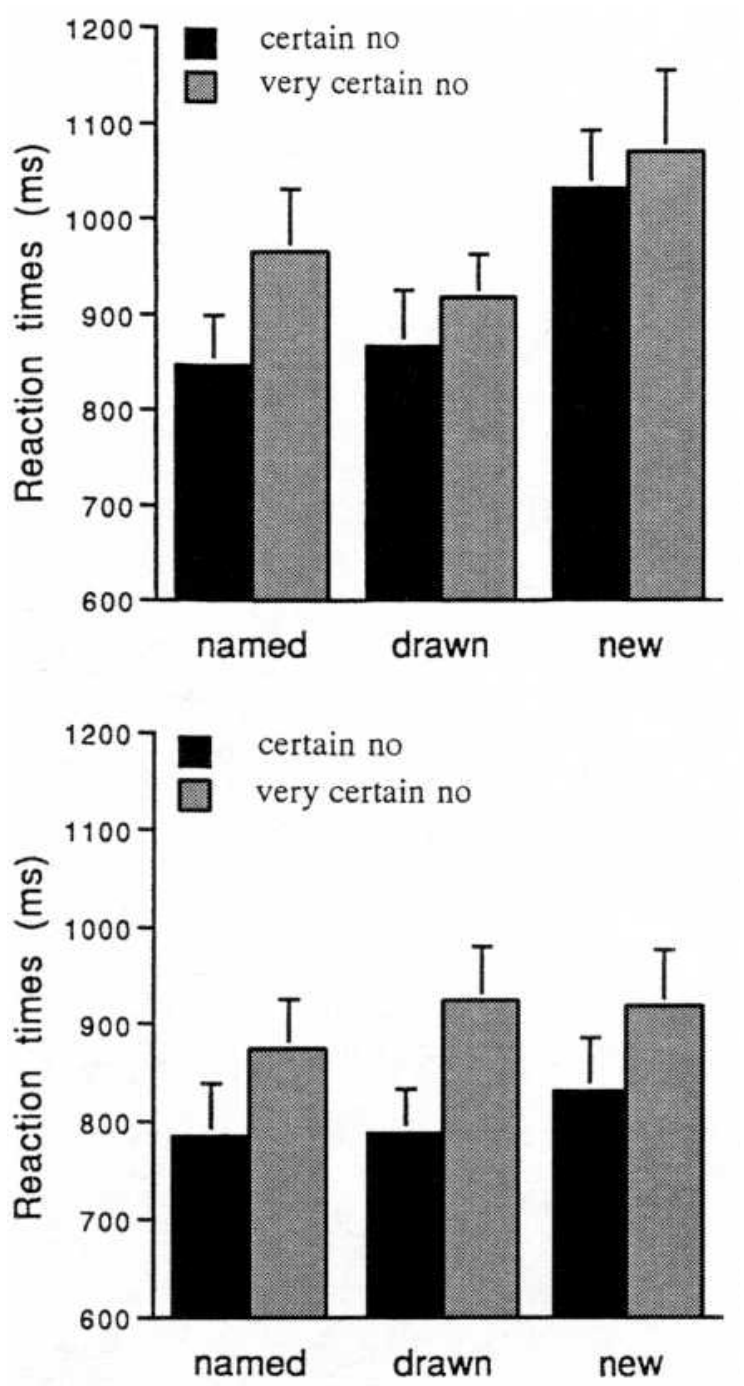

Figure 4. Top panel: Mean reaction times (RTs; and standard errors) across 15 participants who used both certainty levels in Experiment 1 during the test phase to unidentified objects one level prior to identification as a function of response certainty. Speed of "certain no" and "very certain no" responses are shown for fragments of objects that had been named, drawn, or were new. Bottom panel: RTs (and standard errors) across the 14 participants who used both certainty levels in Experiment 1 during the test phase to unidentified objects two levels prior to identification as a function of response certainty. Speed of "certain no" and "very certain no" responses are shown for fragments of objects that had been named, drawn, or were new.

seen in Figure 6 (top panel), ERPs to unidentified objects are generally more negative than those to identified objects: 300-600 ms, $F(2,28)=109.07, p<.00001 ; 600-1,000 \mathrm{~ms}$, $F(2,28)=125.38, p<.00001 ; 1,000-1,300 \mathrm{~ms}, F(2,28)-$ 23.36, $p<.00005$.

For studied pictures, the shift from negativity to positivity is gradual, whereas for new pictures, it is more abrupt. For unidentified pictures, larger amplitudes are observed, at medial leads, over the left than right hemisphere for both 
FRONTAL
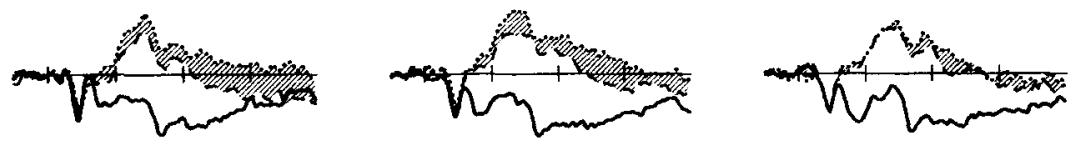

CENTRAL
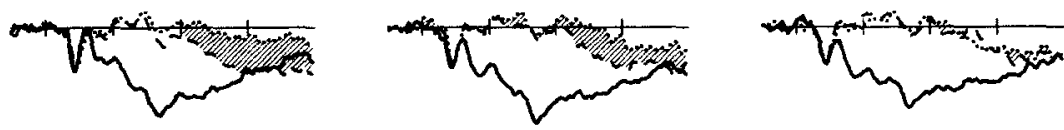

PARIETAL

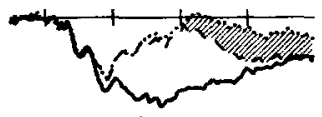

L. FRONTAL
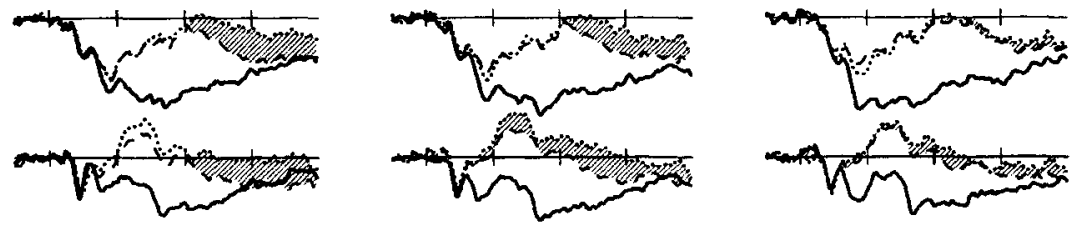

R. FRONTAL
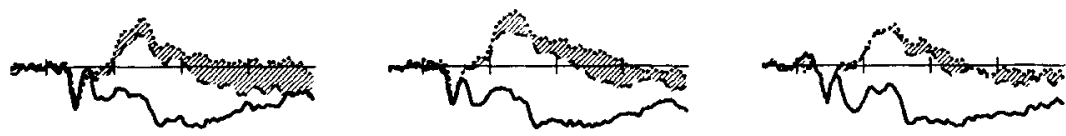

L. CENTRAL

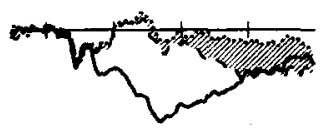

R. CENTRAL
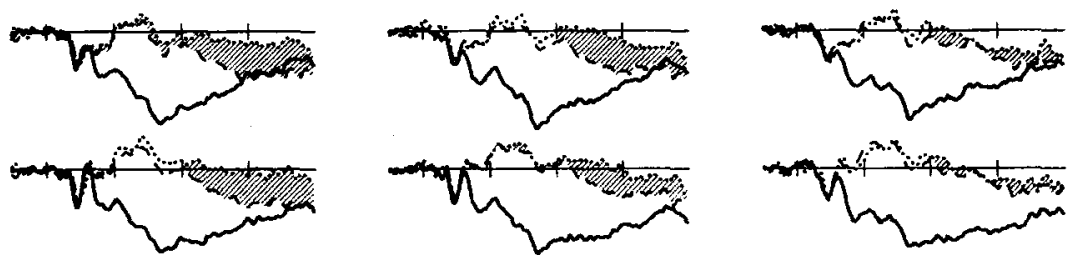

L. PARIETAL
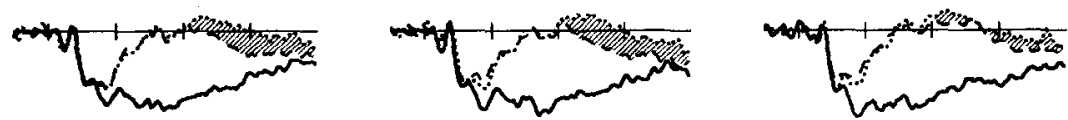

R. PARIETAL
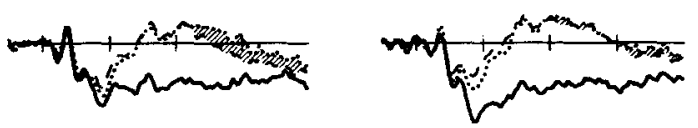

L. OCCIPITAL
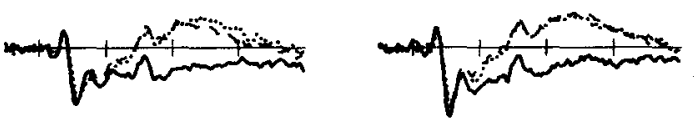

R. OCCIPITAL
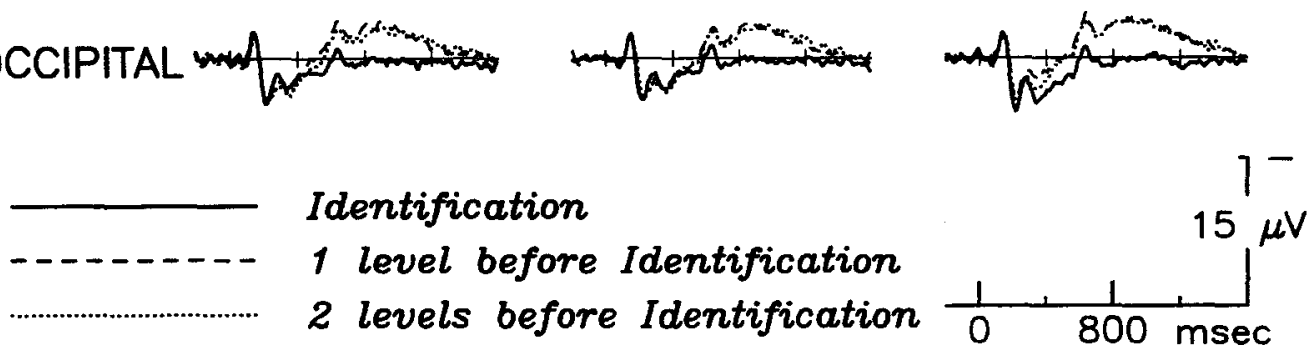

Figure 5. Grand average ( $\mathrm{N}=15)$ event-related potentials to objects upon identification ( 0 ; solid line), one level before ( -1 ; dashed line), and two levels before ( -2 ; dotted line) for objects that had been named, drawn, or were new in Experiment 1. Negativity is up on this and all subsequent figures. L. = left; R. = right.

studied and unstudied pictures. At the moment of identification, no hemispheric asymmetries are observed. For studied pictures, ERP amplitudes at -1 are larger over left than right hemisphere. The difference between -1 and -2 is evident over the left hemisphere, especially in medial and lateral sites.

This pattern of effects was examined further in a subsequent ANOVA on ERPs to unidentified objects at Levels -1 and -2 . 

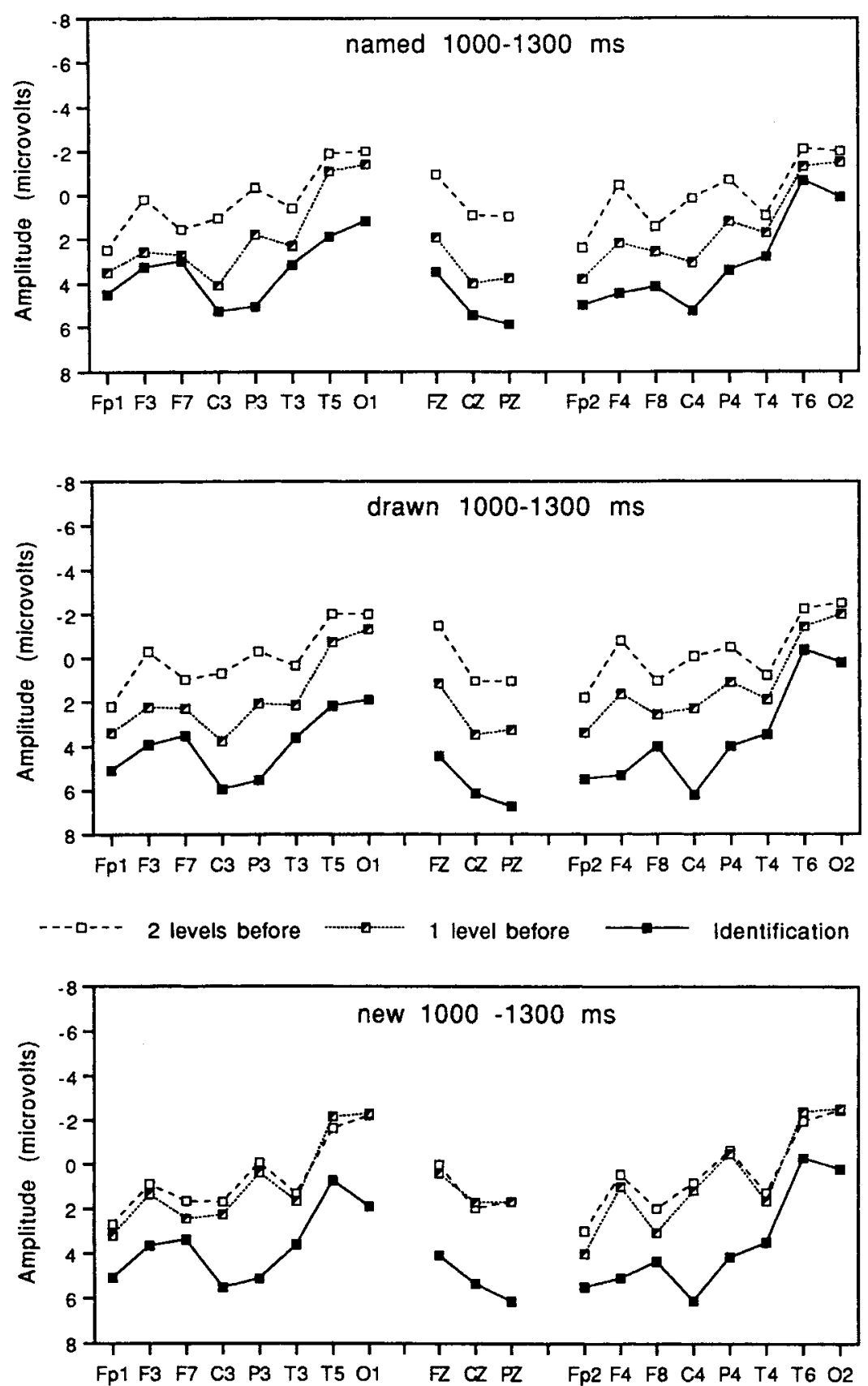

Figure 6. Comparison of the distributions across the scalp of the mean amplitude between 1,000 ms and 1,300 ms for event-related brain potentials at identification ( 0 ; solid line), one level before (-1; dotted line), and two levels before ( -2 ; dashed line) for objects that were named, drawn, or new to the identification phase. Data on the left and right sides of the graph are from over left (odd numbers) and right (even numbers) hemispheres, respectively, and those in the middle are from midline locations, going from front to back. $\mathrm{F}=$ frontal; $\mathrm{C}=$ central; $\mathrm{T}=$ temporal; $\mathrm{P}=$ parietal; $\mathrm{O}=$ occipital.

This analysis revealed no main effect of study at any latency but revealed a main effect of time course between $1,000-1,300 \mathrm{~ms}$, $F(1,14)=33.43, p<.0001$, because of the greater positivity at -1 relative to -2 , as well as a significant interaction of study with time course, $F(2,28)=7.81, p<.002$, reflecting the greater positivity for studied but not for new objects at -1 relative to -2 .
The same pattern of preidentification ERP effects is observed whether the comparison was between drawn and new objects; main effect of identification time course, $F(1$, $14)=22.8, p<.0003$; Study $X$ Time course, $F(1,14)=$ $11.25, p<.005$; or between named and new objects; main effect of identification time course, $F(1,14)=14.30, p<$ 


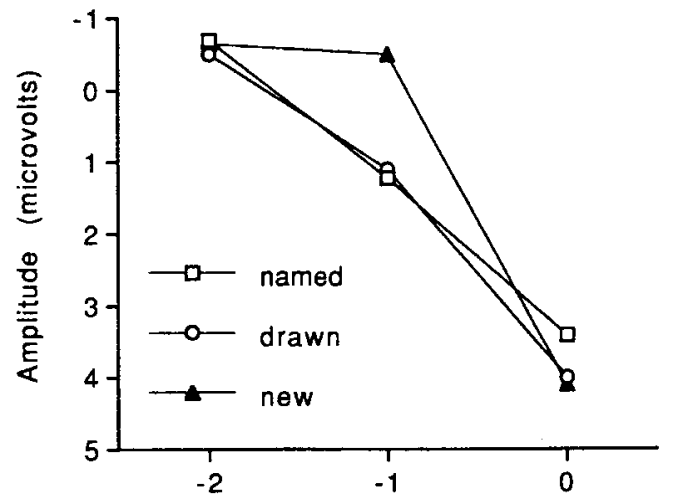

Figure 7. Event-related brain potential amplitude (parietal lead) at $-2,-1$, and 0 for named, drawn, and new objects.

.002 ; Study X Time course, $F(1,14)=13.36, p<.0026$. Compared within a condition, the ERPs at -2 versus -1 were not significantly different for new objects, but they were significantly different for both drawn and named objects $(p<.01)$, being more positive at -1 than -2 . Additional analyses also showed that the effect of study was significant at $-1(p<.04)$ but not at $-2(p<.18)$. At -1 , the ERPs to both drawn and named objects were more positive than new ones, $t(15)=3.121, p<.001$, and $t(15)=$ $2.603, p<.05$, respectively, and did not differ from each other (Figure 7).
There was no effect of study on the latency of the large positivity (around $700 \mathrm{~ms}$ ) elicited upon identification.

Identification $(0)$ versus one level after $(+I)$. As can be seen in Figure 8, the primary difference between ERPs at identification and those after $(+1$, because there were not enough trials for unstudied objects at +2 ) is in the shorter peak latency in the positivity for after; main effect of identification time course, $\mathrm{F}(1,14)=86.43$, $\mathrm{p}<.00001$. Differences in amplitude are relatively small and restricted mostly to posterior sites where +1 ERPs are also somewhat more positive than those at identification; this was revealed in a significant Identification X Anterior-to-Posterior Electrodes interaction for peak amplitude, $\mathrm{F}(2,28)=5.18$, $\mathrm{p}<$ 05 , in a four-way ANOVA with repeated measures, with three levels of study, two levels of identification time course, three levels of anterior-to-posterior electrodes, and five levels of left-to-right electrodes.

Early components. We also examined the effects of prior study and fragmentation on several early components known to be sensitive to physical features of input, including the posterior P100, posterior P200, and anterior P200. The posterior P100 component was measured as the largest amplitude positivity between 50 and $150 \mathrm{~ms}$ relative to a 200-ms prestimulus baseline at seven posterior recording sites. These data were collapsed across identification and subjected to a three-way ANOVA with three levels of study, five levels of fragmentation $(3,4,5,6$, and 8$)$ and seven electrodes (T5, P3, 01, Pz, 02, P4, and T6). Level 2 was not

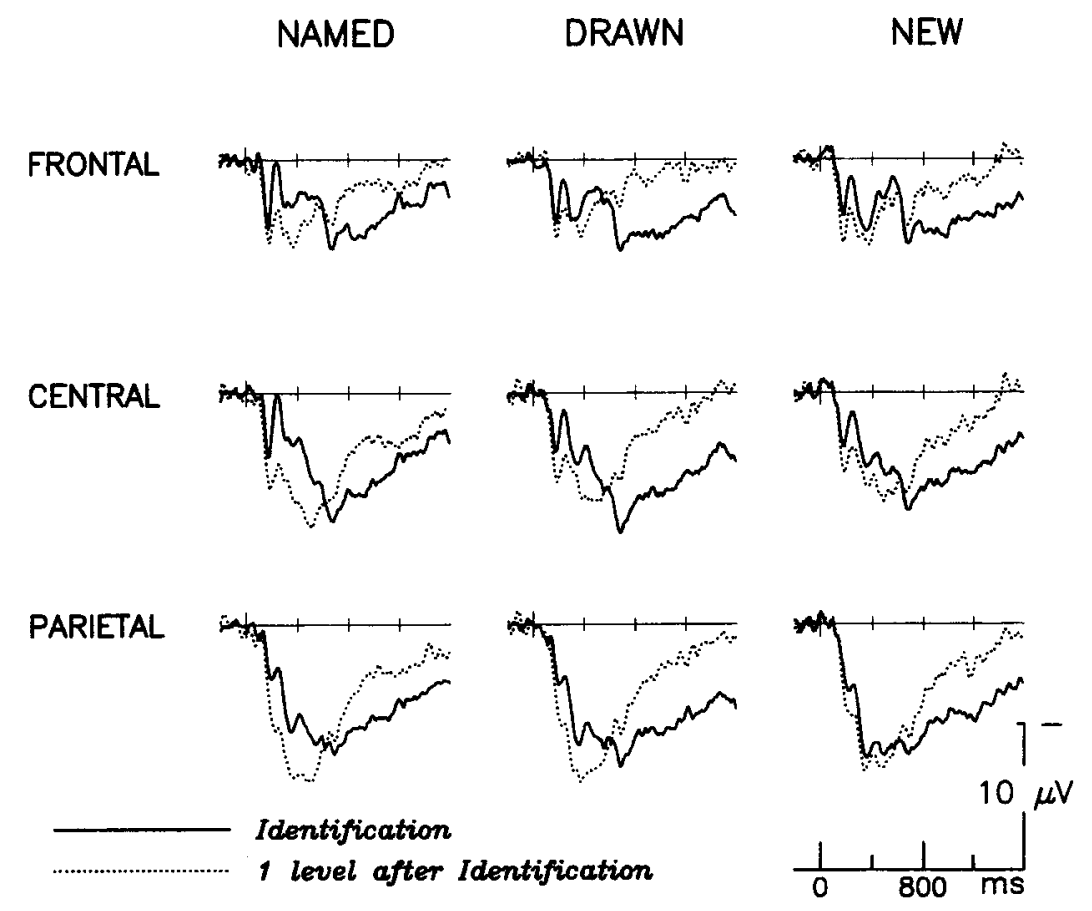

Figure 8. The grand average $(\mathrm{N}=15)$ event-related brain potentials at identification $(0$; solid line $)$ and one level after ( +1 ; dashed line) are compared at three midline recording sites for fragments of objects that had been named, drawn, or were new in Experiment 1. 
included in the analysis because the ERPs were very different from those to subsequent levels. The main effect of fragmentation was significant, $\mathrm{F}(4,56)=3.77$, $\mathrm{p}<.0025$, reflecting the increasingly larger $\mathrm{P} 100$ with fragmentation level (Figure 9).

A similar ANOVA applied to the posterior P200 (measured between 150 and $250 \mathrm{~ms}$ ) also showed a significant effect of fragmentation, $\mathrm{F}(4,56)=21.04$, $\mathrm{p}<.00001$, likewise reflecting an increase in $\mathrm{P} 2$ amplitude from Levels 3 to 8 (Figure 9). The main effect of fragmentation was reliable for both identified, $\mathrm{F}(2,28)=10.75$, $\mathrm{p}<.0001$, and unidentified objects, $\mathrm{F}(2,28)=3.51, \mathrm{p}<.005$. This latency showed the first sign of a study effect in the ERP: Posterior P2 amplitudes were marginally larger to fragments of studied than new objects, $\mathrm{F}(2,28)=3.36, \mathrm{p}<.08$. Subsequent analyses also revealed that the increase in posterior P2 amplitude from Levels 3 to 8 for unidentified objects was more gradual for previously studied than for new objects, $\mathrm{F}(4,56)=3.41, \mathrm{p}<.025$.

A similar ANOVA was performed on the anterior P200 measured at 10 electrode sites (F7, F3, Fz, F4, F8, T3, C3, $\mathrm{Cz}, \mathrm{C} 4$, and T4). Like the posterior P2, the anterior P2 increased in amplitude as the fragmented object approached completeness; main effect of fragmentation, $\mathrm{F}(4,56)=$ 12.07, $\mathrm{p}<.00001$. This pattern held for both identified, $\mathrm{F}(2$, $28)=10.16, \mathrm{p}<.005$, and unidentified objects, $\mathrm{F}(2,28)=$ $3.90, \mathrm{p}<.05$. A reliable effect of study was also found on anterior P2, being larger for studied than new objects, $\mathrm{F}(2$, $28)=6.05, \mathrm{p}<.001$. Subsequent analyses also indicated that for objects that were identified, the effect of fragmenta- tion was more gradual for objects that had been studied than for new ones, $\mathrm{F}(4,56)=3.18, \mathrm{p}<.05$.

\section{Discussion}

Our interest was in how fragments of objects are analyzed to yield the identification of the objects of which they are a part. To that end, we compared the processing of fragments of previously studied objects with those of new objects. Because any given participant was likely to first identify different objects at different levels of fragmentation and any given object was likely to be first identified by different participants at different fragmentation levels, the data were examined relative to the level at which each object was identified.

Not surprisingly, the same number of fragments that were too few to support perceptual completion or identification when presented in isolation were sufficient for successful identification when presented soon after the complete object was first seen. Although any exposure was better than none, an object that had been drawn benefited more than one that had been named. We suggest that the added benefits of drawing are because drawing affords (a) more exposure to partial views of the object, and fragments only provide a partial view, (b) more exposure to various relationships among the parts or fragments of the object, and (c) greater overlap between the analytic processes involved in deconstructing the complete object during the study and attempts to reconstruct it from fragmentary input during the test.

Recent exposure also reduced the time participants took to decide whether or not they could discern an object from the

\section{Frontal Central L. Parietal R. Parietal}
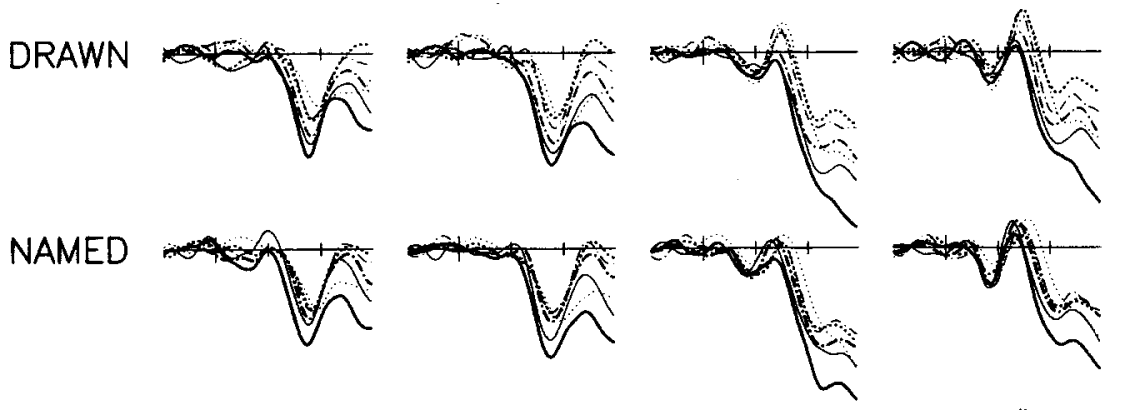

NAMED
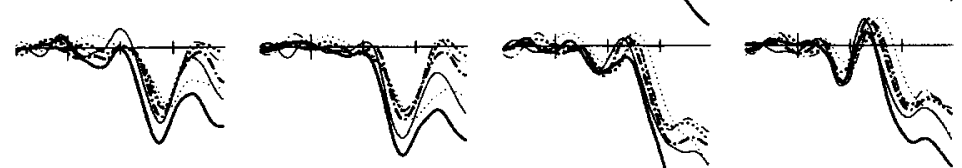

NEW
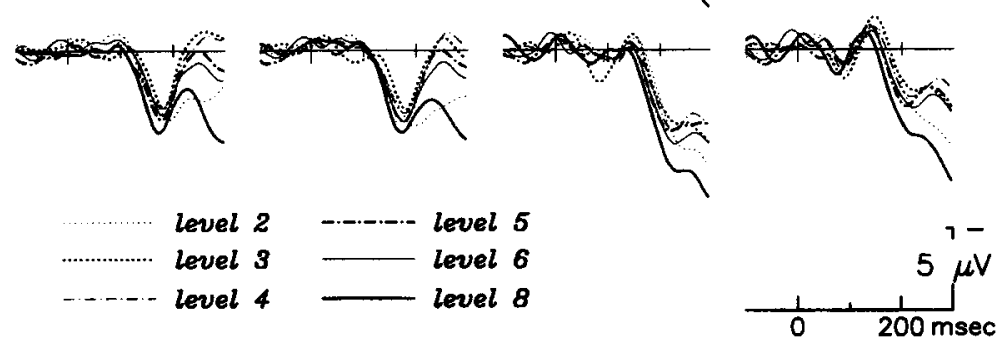

Figure 9. The first $300 \mathrm{~ms}$ of the grand average event-related brain potentials $(\mathrm{N}=15)$ are overlapped for the different fragmentation levels at two midline (frontal and central) and two lateral (left [L.] and right [R.] parietal) sites. 
fragments with any certainty. Not surprisingly, successful identifications were given reliably faster for studied than for new objects, although there was no added benefit from having drawn as opposed to having named them. Note that this pattern of RTs cannot be accounted for in terms of different numbers of input fragments, because the fastest RTs were to drawn objects, which were identified on the basis of fewer fragments than were new objects on the average. $^{1}$

Perhaps more surprisingly, RTs were reliably faster to old than new objects even at the level prior to identification, that is, even when the participants could not identify the object from current visual input. Thus, whether the response was a "yes" as in the case of identification or a "no" as in the case of the prior level, prior exposure was reflected in speeded RTs (relative to those for new objects). Clearly, at least some visual processes were "primed" by prior exposure before the object was overtly identified. We suggest that the episodic memory trace(s) created at study influenced how or how quickly the current visual input was processed. We suggest that this influence is not likely to be conscious or strategic in a psychological sense nor strictly localized to higher level areas (such as inferotemporal regions) in a physiological sense. Among the possibilities, for example, the speeded RTs may have reflected lowered thresholds of activation for neurons in early visual areas such as V 1 and V2, as fMRI and PET data suggest that priming is reflected in reduced activity or greater synchrony between activated regions both within and across levels of the visual system (James, Humphrey, Gati, Menon, \& Goodale, 1999; Martin, Wiggs, Ungerleider, \& Haxby, 1996; Spitzer, Kwong, Kennedy, Rosen, \& Belliveau, 1995). There was some suggestion in the early components of the ERPs that sensory processing is different (more systematic and gradual) for studied than for new objects by around 150-250 ms.

We believe that this pattern of RTs places important constraints on models of visual processing (and object identification). For example, they would seem to rule out a model of identification that requires an exhaustive, serial search of many memory representations. It could have been the case that responses would have been slower to recently studied objects. Such a pattern might have resulted, for instance, if many partially activated representations created during the study needed to be searched serially and exhaustively. This seems not to have been the case; however, prior to identification, it was the studied rather than the unstudied objects that were associated with faster "no" responses.

As expected, RTs also were faster for the more than less certain responses whether or not the object was identified, regardless of prior study. Thus, prior exposure seems to affect processing time and identification performance (most likely although not necessarily implicitly) but does not necessarily affect the confidence with which the associated decision was made.

The most striking change in the ERPs with identification was the presence of a large positivity starting around $300 \mathrm{~ms}$, with an even shorter latency for fragmentation levels after an object was already identified. This positivity was in stark contrast to the large, late negativity in the same time window that characterized the ERPs prior to identification. This is the same ERP pattern that Stuss et al. (1992) observed. Neither at identification nor for fragments thereafter was there any differential effect of study of the ERPs; that is, neither the amplitude nor the latency of the positivity was greater for studied than new objects (but note that there are more input fragments for new than old objects in this comparison).

There was, however, a striking effect of study on the ERPs for objects at the fragmentation level just prior to their overt identification. At this level, neither new nor studied objects were overtly identified, yet they elicited a clearly different pattern of ERPs. For new objects, the ERPs at -1 are like those at the prior level (-2), namely, large and negative. By contrast, for studied objects, the ERPs at -1 are more positive than those at -2; in fact, it appears as if the ERP at -1 contains a smaller and later version of the positivity that characterizes successful identifications. In other words, recently studied objects that were about to be identified overtly at the next fragmentation level elicited a very late positivity (around $800 \mathrm{~ms}$ and following), whereas new objects that also were about to be identified did not.

We believe that this small, late positivity for studied but not quite identified objects is a sign of covert identification. More specifically, we suggest that it reflects the partial match resulting from the convergence of bottom-up and top-down processes, along the lines of Grossberg's (1995) adaptive resonance theory. This matching process compares sensory input with learned expectations (i.e., learned representations in long-term memory of which bottom-up signal patterns led to that higher level pattern in the past). It reinforces and amplifies those features in the bottom-up pattern that are consistent with top-down expectations (memories that were based on prior experience) and suppresses those that are inconsistent. If the bottom-up and top-down influences are such that they continue to reactivate each other (i.e., resonate), a resonance develops that binds spatially distributed features into a stable equilibrium or a synchronous oscillation. Both more fragments (bottom-up) and a prior study episode (top-down) can increase the likelihood of resonance, which is an essential precursor to recognition, according to Grossberg. Within this type of framework, we suggest that the late, small positivity reflects an indirect match (and no direct mismatch) between memory and sensory input; that is, the visual input matches with a part of the memory and does not directly mismatch with any part of the memory; in fact the top-down representation can in combination with the sensory input complete the object. This partial match, however, is not sufficient to support

${ }^{1}$ Although this aspect may confound the effect of prior exposure and number of fragments, we believe that this is not a real problem in the interpretation of either the RT or the ERP data. The RT pattern we observe as a function of study is opposite that one would have predicted if the differences were due simply to different numbers of fragments at one level before identification, for example, for studied versus new objects. Our RT priming effects would be stronger only if the number of fragments was identical in two cases. 
resonance of a duration needed for overt identification, because the bottom-up signal is too weak.

\section{Experiment 2}

The findings of Experiment 1 are intriguing in showing the role of prior experience on visual processing. However, there are alternative explanations for this pattern of results. For example, it may be that the amount of processing and template matching are equivalent for all unidentified pictures, but that people vary in their degree of confidence in the identification. On this account, identification may still be an all-or-none process, and the late positive ERP difference between -1 and -2 is due to greater confidence in the decision at the level immediately prior to identification. Likewise, the difference between ERPs to previously studied versus new objects at -1 may be due to greater confidence for decisions about studied objects. In fact, P300 amplitude has been reported to vary with confidence, with high confidence decisions eliciting larger P300s (e.g., Wilkinson $\&$ Seales, 1978). There were not enough trials in this data set to yield reliable averages to examine whether the ERP effects were different as a function of response certainty.

We thus conducted a second experiment, both to replicate the first and to determine whether the gradual changes in ERPs for studied objects during the identification phase could be attributed to people's response certainty in any way. Specifically, we aimed to test the possibility that the gradual change in the ERP related to identification is due to differential confidence in participants' identifications at various levels and not to the identification process per se.

In order to have enough trials for reliable ERP averages at each level of response certainty, the name condition was dropped and the number of trials in the draw condition was doubled. In addition, another level of fragmentation (Level 7) was added to the six levels used during identification in the previous study. In all other respects, the two experiments were identical.

\section{Method}

\section{Participants}

Eighteen right-handed adults ( 8 women) between 19 and 30 years of age, with no left-handed people in their immediate family and with normal or corrected-to-normal vision were paid \$5 an hour for their participation.

\section{Stimuli}

Stimuli were the same as in Experiment 1, except that each picture was presented at seven instead of six levels of fragmentation.

\section{Electrophysiological Recordings}

All aspects of the electrophysiological recordings were as in Experiment 1.

\section{Procedure}

The procedures were the same in all respects as in Experiment 1 with the exception that all the objects were drawn during the study.

\section{Results}

The main findings of Experiment 1 were replicated overall. Specifically, recently studied objects were identified on the basis of fewer fragments than new objects were, and RTs were faster to studied than to new objects at identification (0), as well as at the level before (-1). As in Experiment 1, the ERPs to new objects were marked by a fairly abrupt shift from negative to positive upon identification, whereas those to recently studied objects showed a more gradual shift, with a late positivity to fragments at -1 . Finally, although response certainty did influence the pattern of ERPs, the late positivity to studied (as opposed to new) objects at -1 was not simply due to differential response certainty; it was present even when response certainty was held constant.

\section{Behavioral Data}

Accuracy. As in Experiment 1, objects that had just been studied were identified on the basis of fewer fragments $(\mathrm{M}=5.0)$ than those that had never been studied $(\mathrm{M}=6.5)$; $\mathrm{t}(17)=15.95, \mathrm{p}<.0001$.

$R T s$. RTs (collapsed across response certainty) to fragments of new and drawn objects before, at, and after identification are shown in Figure 10. A two-way ANOVA

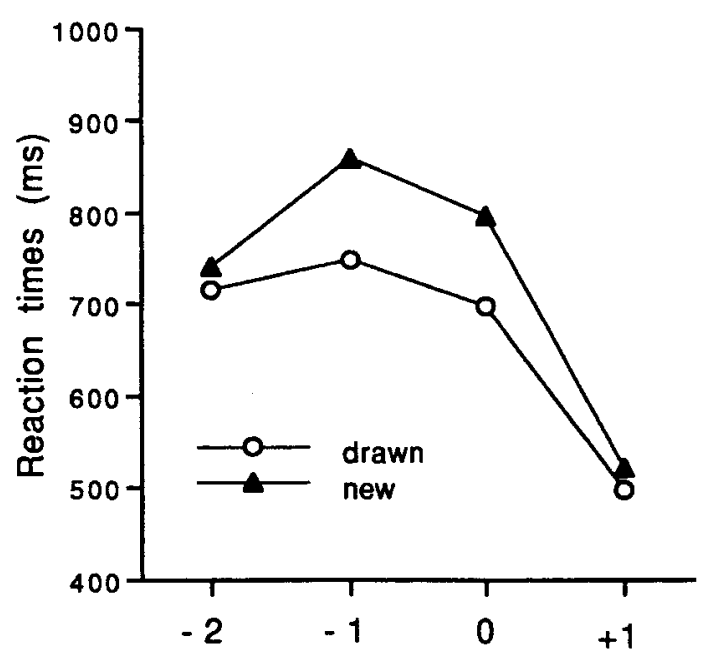

Figure 10. Mean reaction times (RTs) across 18 participants in Experiment 2 to fragments during the identification phase are shown for comparisons to fragments of objects that had been drawn (open circles) versus those that were new (filled triangles) at five different points in the identification sequence: at identification (0), one and two fragmentation levels before $0(-1$ and -2$)$, and at one and two fragmentation levels after $0(+1$ and +2$)$. Note that because measurements are relative to the fragmentation level at which identification occurs, the RT values at each point are collapsed across several fragment levels. 
with repeated measures of two levels of study (drawn and new) and four levels of identification time course $(-2,-1$, 0 , and +1$)$ showed a reliable effect of study, $F(1,17)=$ $21.35, \mathrm{p}<.00025$ : RTs to objects that had been drawn were significantly faster than those to new objects. There was also a significant main effect of identification time course, $\mathrm{F}$ (3, $51)=7.99, \mathrm{p}<.005$. Tukey tests showed that only RTs to fragments at +1 were significantly $(\mathrm{p}<.01)$ faster than those for any other levels. There was also a significant Study X Time Course interaction, $\mathrm{F}(3,51)=3.51, \mathrm{p}<.05$. Simple main effects showed that RTs to previously drawn and new objects differed from each other at $-1, \mathrm{~F}(1,17)=$ $50.96, \mathrm{p}<.00001$, and at $0, \mathrm{~F}(1,17)=14.00, \mathrm{p}<.0025$. In both cases, RTs were faster for drawn than new objects. RTs to drawn and new objects did not differ at all either at -2 or at +1 .

Another two-way ANOVA restricted to the first three stages of time course $(-2,-1$, and 0$)$ revealed only a significant main effect of study, $F(1,17)=20.18$, p < .0005 .

Certainty rates. At -2 , the mean percentages of "very certain no" and "certain no" responses were $54 \%$ and $46 \%$, respectively, $\mathrm{F}(1,17)=1.01, \mathrm{p}=.32$; at -1 , the percentages of "very certain no" and "certain no" were $36 \%$ and $64 \%$, respectively, $\mathrm{F}(1,17)=107.78, \mathrm{p}<.0001$. The Certainty X Study interaction was not significant.

Effects of response certainty. Only 17 of the participants used both levels ("very certain yes" and "certain yes" responses) at 0. As in Experiment 1, at identification RTs were faster for studied than for unstudied pictures, $\mathrm{F}(1,16)=$ $51.10, \mathrm{p}<.0025$, and for "very certain yes" than "certain yes" responses, $\mathrm{F}(1,16)=39.90, \mathrm{p}<.00001$. All 18 participants used both levels ("very certain no" and "certain no" responses) at -1 and -2 . At $-1, \mathrm{RTs}$ were faster for studied than for unstudied objects, $F(1,17)=50.96, p<$ .00001 , and for "very certain no" than "certain no" responses, $\mathrm{F}(1,17)=6.30, \mathrm{p}<.025$. At -2 , RTs were faster for "very certain no" than "certain no" responses, $\mathrm{F}(1,17)=7.93, \mathrm{p}<.025$.

Effects of number of fragments. The relationship between the number of fragments and the speed of the identification judgment was also examined, with the exclusion of Levels 2 and 3 where there were not enough successful identifications. RTs upon identification were subjected to a two-way ANOVA with repeated measures, with two levels of study (drawn and new) and five levels of fragmentation $(4,5,6,7$, and 8$)$. As expected, there was a significant main effect of prior study, $\mathrm{F}(1,17)=30.82$, $\mathrm{p}<$ .00001 , with faster RTs to drawn than new objects. There was a marginal effect of the number of fragments, $F(4,68)=$ $2.57, \mathrm{p}=.08$, with RTs gradually decreasing as the number of fragments increased (Figure 11). There was no reliable Study X Number of Fragments interaction.

\section{ERP Analyses}

First, we describe a comparison of ERPs at -2, -1, and 0 . In a subsequent analysis, ERPs at identification and levels

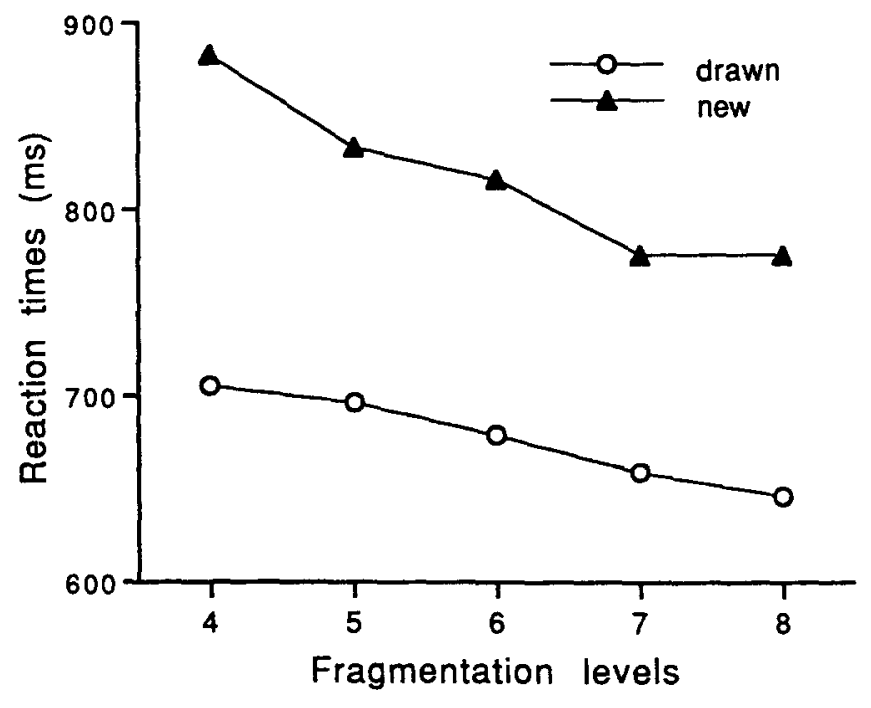

Figure 11. Mean reaction times across 18 participants in Experiment 2 to fragments at each of the ascending levels of fragmentation that had either been drawn during the study phase (open circles) or were unstudied to the test phase (new; closed triangles).

before identification are examined as a function of response certainty.

Levels -2, - 1, and 0. ERPs to unidentified fragments were characterized by a large late negativity, whereas those to identified fragments had a large positivity in the same time window (Figure 12). Mean amplitudes between 300600, 600-1,000, 1,000-1,300, 1,300-1,600 ms were each subjected to a four-way ANOVA with repeated measures with two levels of study (drawn and new), three levels of time course $(-2,-1$, and 0$)$, three levels of anterior-toposterior electrodes (anterior, central, and posterior), and five levels of left-to-right electrodes (left lateral, left medial, central, right medial, and right lateral). The main effect of study was not significant in any measurement window, whereas the main effect of identification was significant in every window: 300-600 ms, $\mathrm{F}(2,32)=32.21$, $\mathrm{p}<.00001$; 600-1,000 ms, $\mathrm{F}(2,32)=35.04, \mathrm{p}<.00001 ; 1,000-1,300$ $\mathrm{ms}, \mathrm{F}(2,32)=3.40, \mathrm{p}<.00001 ;$ and $1,300-1,600 \mathrm{~ms}, \mathrm{~F}(2$, $32)=15.01, p<.00001$. The greater overall negativity for fragments of unidentified $(0.82 \mathrm{uV})$ as opposed to identified $(4.05 \mathrm{uV})$ objects was the confirmed main effect of identification between 300-1,600 ms, $\mathrm{F}(1,16)=42.80$, p < .00001. There was also a significant four-way interaction between study, time course, anterior-to-posterior electrodes, and left-to-right electrodes in each subregion between 300 and 1,600 ms: 300-600 ms, $\mathrm{F}(16,256)=2.61, \mathrm{p}<.025$; 600-1,000 ms, F(16, 256) $=4.17, \mathrm{p}<=.0005 ; 1,000-$ $1,300 \mathrm{~ms}, \mathrm{~F}(16,256)=4.36, \mathrm{p}<.0005 ; 1,300-1,600 \mathrm{~ms}$, $\mathrm{F}(16,256)=3.59, \mathrm{p}<.001$. This interaction reflected a three-step gradation in the amplitude of ERPs at all but occipital sites that was more pronounced for studied than for new objects. As can be seen in Figure 12, the ERPs were most positive at identification, most negative at -2 , and had an intermediate value at -1 . This result is examined further in the following analyses. 
FRONTAL
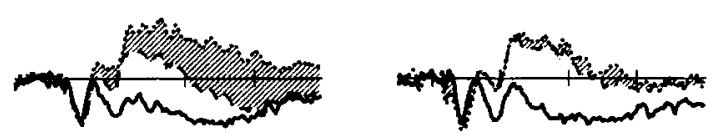

CENTRAL
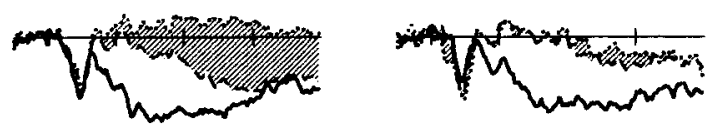

PARIETAL
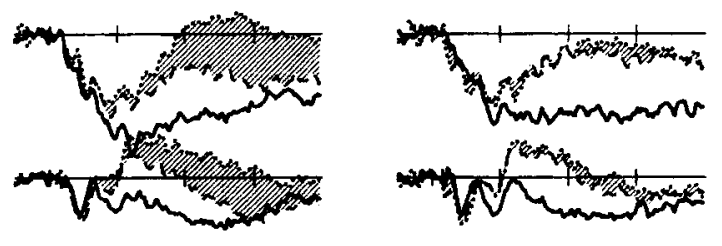

L. FRONTAL

R. FRONTAL
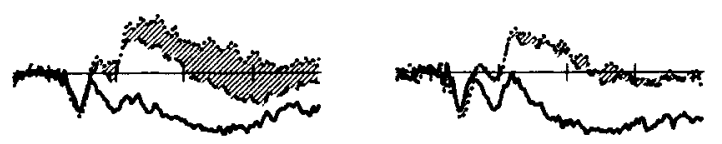

L. CENTRAL
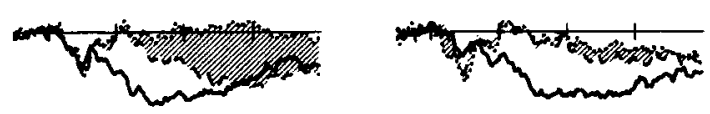

R. CENTRAL
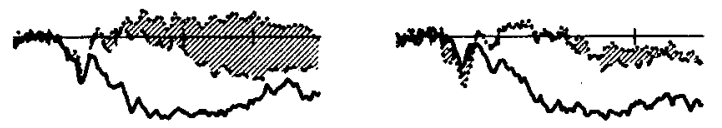

L. PARIETAL
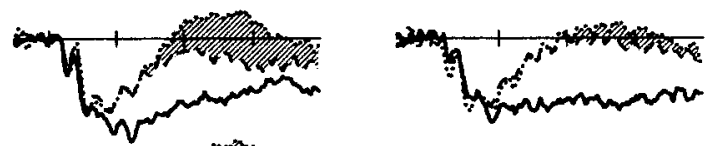

R. PARIETAL
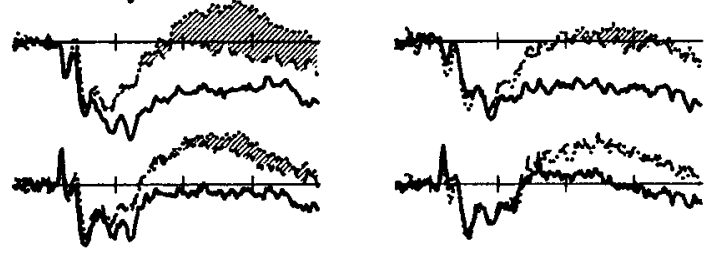

L. OCCIPITAL

R. OCCIPITAL

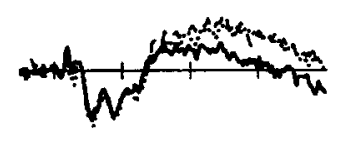

\section{Identification}

- - 1 level before Identification 2 levels before Identification

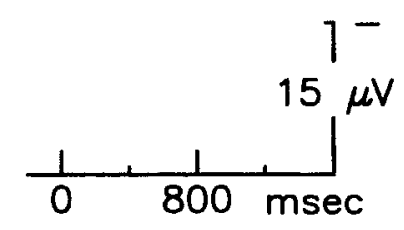

Figure 12. Grand average ( $\mathrm{N}=17)$ event-related brain potentials to objects upon identification ( 0 ; solid line), one level before ( -1 ; dashed line), and two levels before ( -2 ; dotted line) for objects that had been drawn or were new in Experiment 2. 
Level -2 versus Level -1. ERPs to unidentified fragments at -2 and -1 were subjected to two statistical evaluations: The first was based on all the participants' data collapsed across response certainty, and the second was based on the data from the 9 participants who used both levels of response certainty ("very certain no," and "certain no") equally often (so that signal-to-noise ratio for ERPs was the same). These latter analyses will allow a determination of the extent to which the ERP differences at fragmentation levels prior to identification are due to differential response certainty.

A four-way ANOVA with repeated measures was performed with two levels of study (drawn and new), two levels of identification time course ( -1 and -2$)$, three levels of anterior-to-posterior electrodes, and five levels of left-toright electrodes. The main effect of prior study was not significant in any measurement window. Between 300 and $600 \mathrm{~ms}$, there was a significant three-way interaction between study, time course, and left-to-right electrodes, $F(4$, $64)=3.33, p<.05$. A difference between -1 and -2 was found for drawn but not for new objects, which was more pronounced over the left than the right hemisphere sites. This three-way interaction was also significant between $600-1,000 \mathrm{~ms}, F(4,64)=4.03, p<.05$. In this window, although both drawn and new objects showed some difference between -1 and -2, it was noticeably larger for drawn $(2.3 \mathrm{VV})$ than new $(0.8 \mathrm{IN})$ objects. This effect continued between 1,000-1,300 ms as shown by a four-way interaction including the anterior-to-posterior variable as well, $\boldsymbol{F}(8$, $128)=2.73, p<.05$ : Recently drawn objects showed a larger difference between -1 and -2 than did new objects, especially over centroparietal sites of the left hemisphere. In summary, between 300 and 1,600 ms, there was no main effect of study but there was a main effect of identification time course, $F(1,16)=22.75, p<.002$, and a significant interaction between study and time course, $F(1,16)=7.84$, $p<.013$, reflecting the fact that although ERPs were more generally positive at -1 than -2 , the difference was twice as large for drawn than for new objects. The details were pursued in additional analyses that were based on that subset of the participants $(n=9)$ who had enough trials at -2 and -1 at both levels of response certainty.

We performed a five-way ANOVA with repeated measures with two levels of study (drawn and new), two levels of time course (-2 and -1), two levels of response certainty ("very certain no" and "certain no"), three levels of anterior-to-posterior electrodes, and five levels of left-toright electrodes for each of the time windows. Overall, the analyses revealed no main effect of study, significant main effects of identification time course, and significant main effects of response certainty. In addition, however, there were significant two-way interactions between study and identification time course, and identification time course and response certainty, as well as a three-way interaction between study, identification time course, and response certainty.

Because the effects were essentially the same in the various measurement intervals, we reported a detailed analysis on mean amplitudes collapsed across 600-1,300 ms. The significant main effects of identification time course, $F(1,8)=6.22, p<.04$, and response certainty, $F(1$, 8) $=18.59, p<.003$, reflected the generally smaller negativity at -1 than -2 , and smaller negativity for "certain no" than "very certain no" responses, respectively. There was, however, a significant three-way interaction of Study X Identification Time Course X Response Certainty, $F(1,8)=5.12, p<.05$. Further analyses revealed that this reflected no effect of time course for studied or new objects when response certainty was very high, and a striking effect when response certainty was less, but only for studied items (Figure 13). In other words, when participants were merely certain (as opposed to very certain) about their inability to identify objects, then the ERPs to new objects at -2 and -1 were indistinguishable (ns), whereas those to studied items were more positive at -1 than $-2, F(1,8)=45.03, p<$ $.002^{2}$

Identification level. To examine whether ERPs at identification were different as a function of response certainty, we conducted a four-way ANOVA with two levels of study (drawn and new), two levels of response certainty ("very certain yes" and "certain yes"), three levels of anterior-toposterior electrodes, and five levels of left-to-right electrodes. This analysis was restricted to the 12 participants who used both levels of response certainty at identification equally often (this included 6 of the 9 participants in the response certainty analysis for unidentified objects described above). There was no main effect of prior study. There was, however, a significant main effect of response certainty: ERPs to "very certain" identifications were more positive than those to "certain yes" responses from 300 to $1,600 \mathrm{~ms}$, for example, 600-1,000 ms, $F(1,11)=9.53, p<$ 025. In this same time window there was also a significant Study X Response Certainty interaction, $F(1,11)=6.82$, $p<.025$, indicating that the difference between very certain and certain identifications was much larger for new than just drawn objects. The left-to-right electrode variable also was significant between 600 and 1,600 ms, 600-1,000 ms, $F(4$, $44)=32.29, p<.00001 ; 1,000-1,300 \mathrm{~ms}, F(4,44)=$ $30.88, p<.00001 ; 1,300-1,600 \mathrm{~ms}, F(4,44)=23.49, p<$ .0000 . Overall, the positivity associated with identification was large over central and parietal leads, and larger over right than left hemisphere sites. The Study X Response Certainty X Left-to-Right Electrode Interaction was significant, 600-1,000 ms, $F(4,44)=8.59, p<.0025 ; 1,000-$ $1,300 \mathrm{~ms}, F(4,44)=6.91, p<.005 ; 1,300-1,600 \mathrm{~ms}, F(4$, $44)=5.06, p<.025$, generally reflecting the fact that the response certainty effect was largest for new (unstudied) objects over centroparietal sites of the right hemisphere.

Levels $0,+1$, and +2 . The late identification positivity became significantly shorter in latency from 0 to +1 to +2 main effect of identification time course, $F(2,32)=25.20$,

2 We compared ERPs at -1 versus -2 within condition rather than studied versus new within each level because only in the former comparison are the objects (physical stimuli) fairly similar. Given the relatively small numbers of trials in these comparisons, we did not want to confound the effect of study with physical stimulus differences, as would be the case for studied versus new comparisons within a level. 
DRAWN

$$
\begin{aligned}
& \text { Very Certain } \\
& \text { Responses }
\end{aligned}
$$
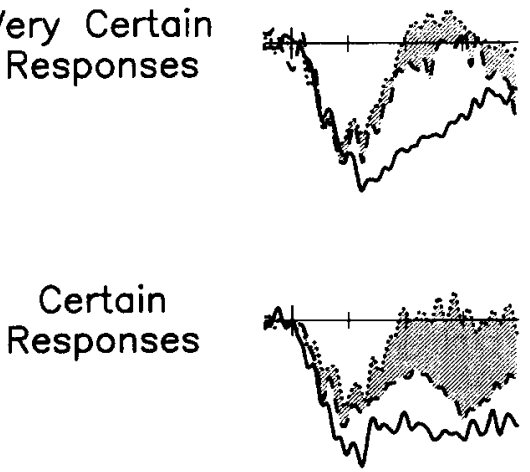

\section{Identification}

-.-. 1 level before Identification 2 levels before Identification
NEW
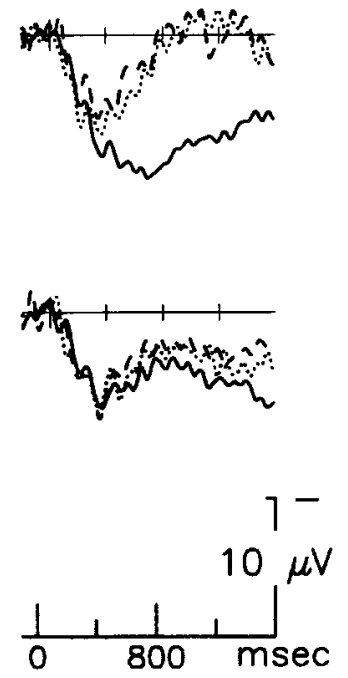

Figure 13. Grand average event-related brain potentials (ERPs) during the identification phase for identified $(n=12)$ and unidentified $(n=9)$ objects sorted according to response certainty (certain and very certain) and prior study (drawn and new). Overlapped in each case are the ERPs to fragments upon identification ( 0 ; solid line), and at one (-1; dashed line) and two (-2; dotted line) fragmentation levels before the identification (0). Note that the ERPs for unidentified objects are based on the 9 participants who used both response certainty levels with similar frequency, whereas the ERPs at identification are based on the 12 participants who used both response certainty levels equally often.

$p<.00001$. The late positivity around $700 \mathrm{~ms}$ was larger in amplitude at +1 , but showed a decrease at $+2, F(2,32)=$ $7.56, p<.0025$. The increase at +1 was larger for new than for drawn objects, $F(2,32)=5.55, p<.001$.

\section{Discussion}

The main findings from Experiment 1 were replicated in Experiment 2: (a) fewer fragments were needed for identification of recently drawn than new objects, (b) identifiability judgments at 0 and -1 were faster for drawn than new objects, and (c) ERPs showed a more gradual shift from negativity to positivity as the number of fragments increased if the objects were old than new. The results of this experiment also eased our concerns about the possible confounds from differential degrees of response certainty on the ERPs to unidentified fragment levels in Experiment 1. Consistent with previous findings (Wilkinson \& Seales, 1978), response certainty did have an effect on identification ERPs. Upon correct identification, the late positivity was larger for responses given with greater certainty, and this effect was larger for studied than new objects. Response certainty effects were also evident on ERPs prior to identification at -1 ; the less certain the response, the larger positivity, more so for studied than new objects. When participants were very certain that they could not identify the objects, then the ERPs were no different at -2 and -1 regardless of whether they had been studied. There was, however, a clear effect of study on the ERPs at -2 versus -1 when the participants gave a merely "certain no" response. At this level of response certainty, ERPs to new objects at -1 and -2 did not differ, whereas those to old objects had small, late positivity at -1 that was intermediate between the negativity at -2 and the large positivity at identification (0). This increase in positivity to unidentified studied objects that was not evident in the ERPs to new objects cannot be attributed to differential response certainty.

As in Experiment 1, nonidentification judgments were characterized by laterally asymmetric potentials. Overall, the potentials over the right hemisphere were more positive than those over the left. At central and posterior sites, the negativity to unidentified fragments was smaller in amplitude over the right than left hemisphere, and the positivity to successful identifications was larger over the right than left hemisphere. The right hemisphere is traditionally considered the Gestalt side of the brain, in part because of its better performance in Gestalt-like tasks, such as perceptual closure of fragmented pictures (McCarthy \& Warrington, 1990). In fact, several investigations with brain-damaged individuals have shown that the two cerebral hemispheres play different roles in object processing. For example, identification of fragmented pictures was more impaired after lesions to right than to left hemisphere (De Renzi \& Spinnler, 1966; 
Viggiano \& Pitzalis, 1998; Warrington \& James, 1967, 1986, 1988; Warrington \& Taylor, 1973). The two hemispheres are also differentially sensitive to prior exposure with the right hemisphere benefiting more than the left. Specifically, Henke, Landis, and Markowitsch (1993) found that objects could be identified on the basis of fewer fragments after the right (but not the left) hemisphere had been exposed to the complete object.

\section{General Discussion}

Hemispheric differences aside, the neural circuits involved in visual object identification respond to some combination of visual input (fragments) and memory in the form of either abstract prototypic representations or operations on specific instances that are effectively prototypic. We believe that some form of the latter is the neural instantiation of what is meant by a template, although for present purposes we need take no stance on its exact representational format. Our take on the current literature leads us to assume that several specific but partial representations may be created for each object; in other words, an object may be represented multiply, albeit with different features coded.

Lesion and single unit investigations with nonhuman animals, functional imaging data from human beings, and behavioral priming studies all suggest that an object (or at least various aspects of an object) is represented throughout the visual system. There appear to be between 25 and 40 different visual areas, each representing somewhat different features or combinations of features of the visual input. Most objects are likely to activate (inhibit or excite) cells in a number of these areas including V 1, V2, V4, inferotemporal cortex, and so forth (Desimone \& Duncan, 1995; Maunsell, 1995; Miller \& Desimone, 1994; Ungerleider, 1995). Evidence indicates that some cells in all of these areas are plastic to some extent; that is, they are sensitive to prior exposure. In other words, these cells reflect a memory of their past firing history in their response to current input. This type of memory is evident in terms of what neurons are sensitive to, how quickly they respond, how long they continue to respond, and the timing of their activations relative to other neurons, among others. Moreover, the visual system is hierarchically organized. Synchronous activation of lower levels results in feedforward activation of higher levels, which in turn feed back onto the lower areas; the flow of information is by no means strictly serial. It thus appears that neurons in several regions within the visual hierarchy fire in concert for an object to be categorized, recognized, and identified correctly (Desimone \& Duncan, 1995; Maunsell, 1995; Miller \& Desimone, 1994; Ungerleider, 1995).

With only minimal input to the visual brain areas, as is the case for Fragmentation Levels 2 and 3, people acknowledge seeing a few incoherent, unidentifiable fragments. "No" responses at these levels were given fairly quickly, in line with the general observation that people do seem to know remarkably quickly (certainly faster than any serial search of memory would allow) when they know absolutely nothing about something.
ERPs at this same level are characterized primarily by early sensory components such as the $\mathrm{Pl}, \mathrm{N} 1$, and $\mathrm{P} 2$ (within the first $200 \mathrm{~ms}$ or so after stimulus onset), emanating from the lower visual areas. These components are known to be sensitive to sensory processing and attentional manipulations. The Pl component, for instance, has been linked to visuospatial attention in general and sensory gating in particular (e.g., Heinze et al., 1994). Its amplitude is much larger for items in the center of an attentional spotlight than for items that are ignored or occur in the periphery. In the present experiments, both the $\mathrm{P} 1$ and the $\mathrm{P} 2$ components show a systematic sensitivity to the number of visual fragments: The more fragments, the larger and the sharper these potentials are and the earlier they peak. At Level 2, the early potentials are quite late and very broad relative to those at lower fragmentation levels. We suggest that when the early levels of the visual system do not get much coherent input (as at Level 2), the signal is not likely to travel as far in the visual recognition hierarchy. Initially, this leads to diffuse, asynchronous activation, and as a consequence to poor local inhibition, diffuse feedforward activity, and thus very little, if any, coherent feedback activity. Perhaps this is why detection is without any real sense of familiarity and is associated with an extremely quick "no" response. It is at times like these that people realize both that they saw something and that no amount of "hard thinking" is going to reveal exactly what it was.

With more input, the initial visual signal (ERP) is larger in amplitude, earlier in latency, and more peaked. Perhaps having more coherent pieces of an object available effectively draws attention to its location. In any case, we take these changes in the early sensory components with increased numbers of fragments to mean that the visual input is sufficient to activate many cells in lower visual areas (V 1 and V2) similarly, and in concert. The greater peakedness of these sensory components is consistent with greater synchrony among the firing of cells sensitive to the same aspects of the input. The neural consequences may be a coherent response for activating higher levels, more effective inhibition of cells with different sensitivities at the same level in the visual hierarchy, and greater likelihood of more effective feedback from higher visual areas. Possible consequences of this could be finer tuning or continued activation of the circuits in the lower visual centers that originated the activity beyond the actual presence of the eliciting stimulus. We suggest that something like this may be the state of affairs during the processing of Fragmentation Levels 4 and up.

It is starting at these intermediate (3-5) levels of fragmentation that our volunteers began to be successful at identifying objects. Among these intermediate levels, it has been suggested that Level 4 is somehow special (Snodgrass \& Feenan, 1990). First, because the perceptual processes engaged by fragments at this level are more likely to lead to their successful identification as an object on the spot. Second, because exposure to Level 4 leads to greater long-term processing benefits than exposure to other levels (including the complete figure). The special quality of this level has been related to the notion of perceptual closure and 
in turn with the moment of identification. Presumably it is at these intermediate levels of fragmentation that visual input is sufficient to support a successful negotiation between the input from the retina, the prior information, and the verbal output system. The outcome of such a settling process in the visual system is a conscious feeling of knowing what object the fragments depict (probably whether or not it was recently seen) and, given the task demands, the ability to find its name.

Theoretical concerns with the notion of perceptual closure notwithstanding, when they first walked in the door, our volunteers could scarcely have identified any object at Fragmentation Level 2, perhaps only one or two more at Level 3, and about a third of them at Level 4. Thus, it is a tribute to the short-term plasticity of the human visual system that after a single exposure to the complete object (named or drawn), significantly more of these objects could be identified at intermediate fragmentation levels in the ascending sequence. In our data, the impact of a single naming or drawing exposure was evident in the fewer numbers of fragments needed to identify the object correctly, shorter identification times, and the pattern of the brain electrical activity associated with the processing and decision making leading to these perceptions, cognitions, behaviors, and memories of them.

In both experiments, identification processes were influenced by prior study. Identification thresholds were lower for objects that had been studied than for those that had not. Having named or drawn the object before was worth about one fragment level. Likewise, identification times were faster for the fragments of previously seen objects. This is not particularly surprising; the results of many studies attest to the facilitory role of learning (Leeper, 1935), training (Gollin, 1960), past experience (Rock, 1975), perceptual priming (Snodgrass \& Corwin, 1988), or priming on the identification of fragmented pictures (Danzinger \& Salthouse, 1978; Henke et al., 1993; Parkin \& Russo, 1990; Roediger \& McDermott, 1993; Salthouse \& Prill, 1988; Snodgrass \& Feenan, 1990; Vokey, Baker, Hayman, \& Jacoby, 1986; Whitfield \& Elias, 1992; Whitfield, Elias, \& Dore, 1991; Wippich et al., 1991). Our results, however, further show that a single 1- to 2-s episode of this sort also influences unsuccessful visual processing of identified objects as well. Surprisingly, we observed that people were faster to respond "no I don't know what object these fragments represent" at the level just before overt identification if they had seen the object before. So the eye-mind-hand combination is affected by the prior study episode, even when the overt identification system remains stumped.

Effects of prior study were also evident in the scalprecorded ERPs. For the first $100 \mathrm{~ms}$, ERPs were sensitive only to bottom-up visual information (i.e., number of fragments). However, between 170 and $250 \mathrm{~ms}$, ERPs reflected not only the number of fragments but also the prior exposure. Thereafter, the pattern of ERPs depends primarily on whether or not the fragments remained unidentified. With successful identification the ERP went positive around 250-300 ms for over half a second. If rendered with high certainty, this broadly distributed, late positivity was equally large for studied and new objects. Otherwise (i.e., for somewhat less certain identifications), this positivity was slightly smaller for drawn objects and much smaller for new objects. This late positivity falls in the class of potentials generally called the P300, P3b, or late positive complex, typically elicited by binary decisions, recognitions, and identification judgments in a number of different experimental paradigms (e.g., Donchin \& Coles, 1988; Verleger, 1988). Within a signal-detection tasks, the amplitude of this positivity increases monotonically with increases in the a posteriori likelihood that a signal was presented.

In our experiments, the P300 to drawn objects was more peaked than to new objects, probably because of greater variability in decision and RTs for the latter. There was, however, no observable study effect on P300 latency. Whereas we might have expected P300s to mirror RTs and be of shorter latency for studied than new objects at identification, this was not the case. However, by comparing the effects of study on identification (collapsed across fragment levels), we may have neutralized the faster processing of studied items by the slowed processing due to fewer fragments. This possibility seems all the more likely given that the late positivity peaked earlier once a set of fragments had been identified accurately, (e.g., for all fragmentation levels postidentification). This pattern would be expected from the literature showing that P300 latency varies with the complexity of the categorization decision (e.g., Kutas, McCarthy, \& Donchin, 1977).

The most novel and noteworthy finding of the present set of experiments is that prior exposure has an effect on visual processing at the level just prior to overt identification. The ERPs to unidentified objects at -1 appear to be a hybrid of the processes for unidentified (mostly negative) and identified (mostly positive) objects; that is, riding on top of the negativity is a late positivity similar albeit smaller and much later than that triggered by overt identifications. Under the circumstances this seems to be a good candidate for a form of "covert" identification. We return to this issue later.

As noted in the introduction, some descriptions of object identification imply that identification is an abrupt, Gestaltlike process, that is, that the processes of identification are qualitatively different from those preceding it. By providing a window on aggregates of neurons functioning in real time, ERPs allow us to see how accurate this view is. In broad stroke, identified and unidentified objects did elicit qualitatively different ERPs: Unidentified objects were associated with a mostly negative response, whereas the response at identification was mostly positive, especially when poeple responded with high certainty. On the other hand, if perceptual closure merely reflected the final moment of a more gradual process, such as the ongoing attempts to coordinate and combine activated visual representations with each other and with more semantic and verbal representations so as to yield the correct overt identification, then we might expect to see the buildup of this evidence in the ERPs even before the stimulus is actually identified. Thus, the ERP data suggest that identification is all or none when there is no recent information about the item available (i.e., for new pictures), 
but more gradual for items that do have recently activated representations (i.e., for named and drawn pictures).

An obvious sign of a qualitative difference in effects from specific as opposed to generic visual memories is the late positivity to previously studied (as opposed to new) objects at one fragmentation level before identification. Around 800 ms or so (approximately $200 \mathrm{~ms}$ before any overt response), there was a clear sign of which fragments were from old objects and which were from new; at this point, the ERPs to old fragments were more positive for the duration of the recording epoch (approximately $400 \mathrm{~ms}$ or so). We take this as some sign of covert identification; ${ }^{3}$ it seems to be contingent upon the existence of a recently formed memory trace, for it is not present for new objects.

Structure is either in the stimulus or in memory. Insofar as the two agree uniquely there is a clarity of perception and reinforcement of the memory, making it easier to reinstantiate and identify (i.e., the memory is reinforced by the stimulus). The less similar the stimulus and any memory, the more partial memory patterns are likely to be activated, thereby leading to more competition for any definitive response. We view perception as adding memory to the stimulus. We find that the nature of the memory matters and that experience is the mother of memories, whether declarable or not.

\footnotetext{
3 The hypothesis may be advanced that the difference between studied and unstudied at -1 is merely a fragmentation effect. Let us assume that more fragmented versions of the stimulus are associated with more negativity, certainly Level 2 fragments are quite negative. Also, suppose that for studied pictures, identification $(0)$ occurs at Level 5,-1 at Level 4, and -2 at Level 3. Then, on the average for unstudied pictures, 0 occurs at Level 6, -1 at Level 5, and -2 at Level 4 . If the negativity goes with more fragmentation, we should have found more negativity for studied than for unstudied across all three levels $(0,-1$, and -2$)$. However, we did not find it. Instead we found that at -1 , studied items were more positive than unstudied items and included more highly fragmented items than unstudied ones. Thus, even if fragmentation plays some role, it cannot completely explain the effect we call covert identification.
}

\section{References}

Biederman, I. (1987). Recognition-by-components: A theory of human image understanding. Psychological Review, 94, 115147.

Brown, R., \& MacNeill, D. (1966). The "tip of tongue" phenomenon. Journal of Verbal Learning and Verbal Behavior, 5, 325-337.

Craik, F. I. M., \& Lockhart, R. S. (1972). Levels of processing: A framework for memory research. Journal of Verbal Learning and Verbal Behavior, 11, 671-684.

Danzinger, W. L., \& Salthouse, T. A. (1978). Age and the perception of incomplete figures. Experimental A ging Research, 4, 67-80.

De Renzi, E., \& Spinnler, H. (1966). Visual recognition in patients with unilateral cerebral disease. Journal of Nervous and Mental Diseases, 142, 515-525.

Desimone, R., \& Duncan, J. (1995). Neural mechanisms of selective visual attention. Annual Review of Neuroscience, 18, 193-222.

Donchin, E., \& Coles, M. G. H. (1988). Is the P300 component a manifestation of context updating? Behavioral and Brain Sciences, 11, 357-428.

Gollin, E. S. (1960). Developmental studies of visual recognition of incomplete objects. Perceptual and Motor Skills, 11, 289-298.

Grossberg, S. (1995). The attentive brain. American Scientist, 83, 438-449.

Heinze, H. J., Mangun, G. R., Burchert, W., Hinrichs, H., Scholz, M., Munte, T. F., Gos, A., Scherg, M., Johannes, S., Hundeshagen, H., Gazzaniga, M. S., \& Hillyard, S. A. (1994, Dec.). Combined spatial and temporal imaging of brain activity during visual selective attention in humans. Nature, 372, 543-546.

Henke, K., Landis, T., \& Markowitsch, H. J. (1993). Subliminal perception of pictures in the right hemisphere. Consciousness and Cognition, 2, 225-236.

Humphreys, G. W., \& Bruce, V. (1989). Visual cognition: Computational, experimental and neuropsychological perspectives. Hillsdale, NJ: Erlbaum.

Humphreys, G. W., \& Riddoch, M. J. (1987). Visual object processing: A cognitive neuropsychological approach. Hillsdale, NJ: Erlbaum.

James, T. W., Humphrey, G. K., Gati, J. S., Menon, R. S., \& Goodale, M. A. (1999). Repetition priming and the time course of object recognition: An fMRI study. NeuroReport, 10, 10191023.

Jasper, H. H. (1958). The ten-twenty electrode system of the International Federation. Electroencephalography and Clinical Neurophysiology, 10, 371-375.

Koffka, K. (1935). Principles of Gestalt psychology. New York: Harcourt Brace.

Kutas, M., McCarthy, G., \& Donchin, E. (1977). Augmenting mental chronometry: P300 as an index of stimulus evaluation time. Science, 197, 792-795.

Leeper, R. (1935). A study of a neglected portion of the field of leaming. The development of sensory organization. Journal of Genetic Psychology, 46, 41-75.

Martin, A., Wiggs, C. L., Ungerleider, L. G., \& Haxby, J. V (1996). Neural correlates of category-specific knowledge. Nature, 379, 649-652.

Maunsell, J. H. R. (1995). The brain's visual world: Representation of visual target in cerebral cortex. Science, 270, 764-768.

McCarthy, R. A., \& Warrington, E. K. (1990). Cognitive neuropsychology: A clinical introduction. Orlando, FL: Academic Press.

Miller, E. K., \& Desimone, R. (1994). Parallel neuronal mechanisms for short-term memory. Science, 263, 520-522.

Mooney, C. M. (1954). A factorial study of closure. Canadian Journal of Psychology, 8, 51-61.

Nelson, T. O., Gerler, D., \& Narens, L. (1984). Accuracy of feeling-of-knowing judgments for predicting perceptual identification and learning. Journal of Experimental Psychology: General, 113, 282-300.

Parkin, A. J., \& Russo, R. (1990). Implicit and explicit memory and the automatic/effortful distinction. European Journal of Cognitive Psychology, 2, 71-80.

Rock, 1. (1975). Introduction to perception. New York: Macmillan.

Roediger, H. L., III, \& McDermott, K. B. (1993). Implicit memory in normal human subjects. In F. Boller \& J. Grafman (Eds.), Handbook of neuropsychology (Vol. 8, pp. 63-131). Amsterdam: Elsevier.

Salthouse, T. A., \& Prill, K. (1988). Effects of aging on perceptual closure. American Journal of Psychology, 101, 217-238.

Snodgrass, J. G., \& Corwin, J. (1988). Perceptual identification 
thresholds for 150 fragmented pictures from Snodgrass and Vanderwart pictures set. Perceptual and Motor Skills, 67, 3-36.

Snodgrass, J. G., \& Feenan, K. (1990). Priming effects in picture fragment completion: Support for the perceptual closure hypothesis. Journal of Experimental Psychology: General, 119, 276296.

Snodgrass, J. G., Smith, B., Feenan, K., \& Corwin, J. (1987). Fragmenting pictures on the Apple Macintosh computer for experimental and clinical applications. Behavior Research Methods, Instruments, \& Computers, 19, 270-274.

Snodgrass, J. G., \& Vanderwart, M. (1980). A standardized set of 260 pictures: Norms for naming agreement, familiarity, and visual complexity. Journal of Experimental Psychology: Human Learning and Memory, 6, 174-215.

Spitzer, M., Kwong, K. K., Kennedy, W., Rosen, B. R., \& Belliveau, J. W. (1995). Category-specific brain activation in FMRI during picture naming. NeuroReport, 6, 2109-2112.

Stuss, D. T., Picton, T. W., Cerri, A. M., Leech, E. E., \& Stethem, L. (1992). Perceptual closure and object identification: Electrophysiological responses to incomplete pictures. Brain and Cognition, 19, 253-266.

Treisman, A. (1992). Perceiving and reperceiving objects. American Psychologist, 47, 862-875.

Ungerleider, L. G. (1995). Functional brain imaging studies of cortical mechanisms for memory. Science, 270, 769-775.

Valentine, T., \& Moore, V. (1995). Naming faces: The effects of facial distinctiveness and surname frequency. Quarterly Journal of Experimental Psychology: Human Experimental Psychology, 48(A), 849-878.

Verleger, R. (1988). Event-related potentials and cognition: A critique of the context updating hypothesis and an alternative interpretation of P3. Behavioral and Brain Sciences, 11, 343427.

Viggiano, M. P, \& Pitzalis, S. (1998). Identification of fragmented pictures in brain-damaged patients. Applied Neuropsychology, 5, 93-99.
Vokey, J. R., Baker, J. G., Hayman, G., \& Jacoby, L. L. (1986). Perceptual identification of visually degraded stimuli. Behavioral Research Methods, Instruments, and Computers, 18, 1-9.

Warren, C. E. J., \& Morton, J. (1982). The effects of priming on picture recognition. British Journal of Psychology, 73, 117-130.

Warrington, E. K., \& James, M. (1967). Disorders of visual perception in patients with localised cerebral lesions. Neuropsychologia, S, 253-266.

Warrington, E. K., \& James, M. (1986). Visual object recognition in patients with right-hemisphere lesion: Axes or features? Perception, 15, 355-366.

Warrington, E. K., \& James, M. (1988). Visual apperceptive agnosia: A clinico-anatomical study of three cases. Cortex, 24, 12-32.

Warrington, E. K., \& Taylor, A. M. (1973). The contribution of the right parietal lobe to object recognition. Cortex, 9, 152-164.

Whitfield, K. E., \& Elias, J. W. (1992). Age cohort differences in the ability to perform closure on degraded figures. Experimental A ging Research, 18, 67-73.

Whitfield, K. E., Elias, J. W., \& Dore, G. (1991). An analysis of task difficulty using the visually degraded stimuli task. Perceptual Motor Skills, 72, 1047-1057.

Wilkinson, R. T., \& Seales, D. M. (1978). EEG event-related potentials and detection. Biological Psychology, 7, 13-28.

Wippich, W., Mecklenbrauker, S., Wentura, E., \& Srumpel, S. (1991). Implizites Gedachtnis bei Vorschulkindern: Eine Prozessanalyse beim Wiederholten Benennen von Bildern [Implicit memory in preschool children: A process analysis through repeated naming of pictures]. Schweizerische Zeitschrift fur Psychologie, 50, 119-128.

Received November, 19, 1997

Revision received July 6, 1999 Accepted July 9, 1999 